\title{
Dihydrolycorine Attenuates Cardiac Fibrosis and Dysfunction by Downregulating Runx1 following Myocardial Infarction
}

\author{
Tingjuan Ni, ${ }^{1}$ Xingxiao Huang, ${ }^{2}$ Sunlei Pan, ${ }^{3}$ and Zhongqiu Lu $\mathbb{D}^{1}$ \\ ${ }^{1}$ Department of Emergency Intensive Care Unit, The First Affiliated Hospital, Wenzhou Medical University, Wenzhou, \\ Zhejiang, China \\ ${ }^{2}$ Department of Cardiology, Zhejiang University, Hangzhou, Zhejiang, China \\ ${ }^{3}$ Department of Coronary Care Unit, The First Affiliated Hospital, Wenzhou Medical University, Wenzhou, Zhejiang, China
}

Correspondence should be addressed to Zhongqiu Lu; lzq_640815@163.com

Received 23 April 2021; Revised 18 July 2021; Accepted 24 September 2021; Published 23 October 2021

Academic Editor: Sanjay Misra

Copyright (c) 2021 Tingjuan Ni et al. This is an open access article distributed under the Creative Commons Attribution License, which permits unrestricted use, distribution, and reproduction in any medium, provided the original work is properly cited.

In spite of early interventions to treat acute myocardial infarction (MI), the occurrence of adverse cardiac remodeling following heart failure due to acute MI remains a clinical challenge. Thus, there is an increasing demand for the development of novel therapeutic agents capable of inhibiting the development of pathological ventricular remodeling. RNA-seq data analysis of acute MI rat models from GEO revealed that Runxl was the most differentially expressed MI-related gene. In this study, we demonstrated that increased Runx1 expression under pathological conditions results in decreased cardiac contractile function. We identified dihydrolycorine, an alkaloid lycorine, as a promising inhibitor of Runx1. Our results showed that treatment with this drug could prevent adverse cardiac remodeling, as indicated by the downregulation of fibrotic genes using western blotting (collagen I, TGF $\beta$, and $\mathrm{p}$-smad3), downregulation of the apoptosis gene Bax, upregulation of the apoptosis gene Bcl-2, and improved cardiac functions, such as LVEF, LVSF, LVESD, and LVEDD. Additionally, dihydrolycorine treatment could rescue cardiomyocyte hypertrophy as demonstrated by wheat germ agglutinin staining, increased expression levels of the punctuate gap junction protein connexin 43, and decreased $\alpha$-SMA expression, resulting in cardiomyocyte fibrosis in immunofluorescence staining. Molecular docking, binding modeling, and pull-down assays were used to identify potential dihydrolycorine-binding sites in Runx1. When Ad-sh-Runx1 was transfected into hypoxia-cardiomyocytes or injected into the hearts of MI rats, the cardioprotective effects of dihydrolycorine were abolished, and the normal electrophysiological activity of cardiomyocytes was disrupted. Taken together, the results of the present study indicate that dihydrolycorine may inhibit adverse cardiac remodeling after MI through the reduction of Runx1, suggesting that dihydrolycorine-mediated-Runxl regulation might represent a novel therapeutic approach for adverse cardiac remodeling after MI.

\section{Introduction}

Adverse cardiac remodeling after myocardial infarction (MI) results in the development of heart failure, which is a leading cause of high mortality rates $[1,2]$. Acute coronary artery blockage after MI can lead to the cardiomyocyte death and eccentric hypertrophy of surviving cardiomyocytes and initiate a reparative process involving the deposition of fibrillar collagens in infarcted and noninfarcted areas. This subsequently causes myocardial fibrosis, which induces harmful cardiac remodeling and cardiac dysfunction [3-5].
Notably, no specific medication exists to reverse adverse cardiac remodeling.

Dihydrolycorine $\left(\mathrm{C}_{16} \mathrm{H}_{19} \mathrm{NO}_{4}\right)$, an alkaloid lycorine, is obtained by contact hydrogenation from lycoris, a derivative of the Lycoraceae family member pyrrophenthridine [6]. It has diverse biological functions, including antitumor [7-9], neuroprotective [10], antiosteoporosis [11], antiviral [12], and anti-inflammatory [13] functions, as reported by various clinical studies. Recently, for the first time, dihydrolycorine was demonstrated to exhibit cardioprotective effects [14]. However, the possible antifibrotic effects of this drug after 


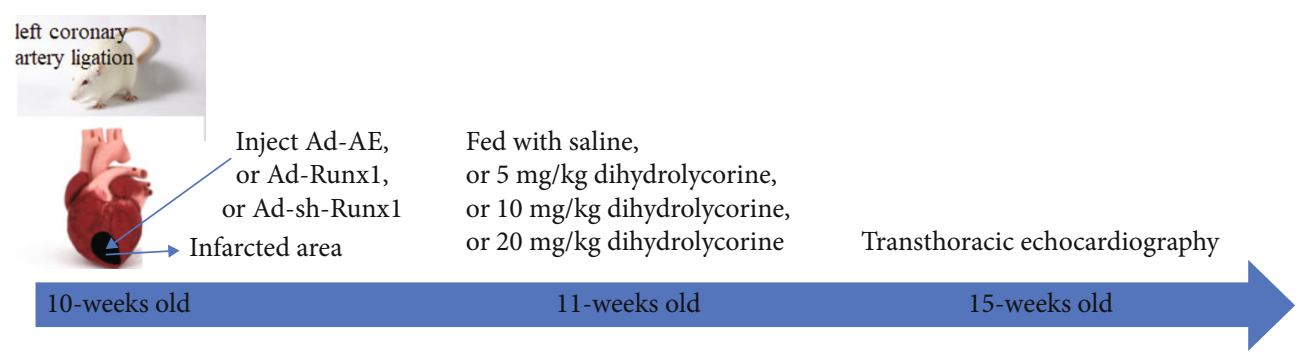

Figure 1: The schematic details on a temporal scale about the animal experiments.

MI should be explored, and the underlying molecular mechanisms involved in cardiac fibrosis should be elucidated.

Runt-related transcription factor-1 (Runx1), a member of the Runt-related (RUNX) family (Runx1, Runx2, and Runx3), possesses a conserved 128 aa region that mediates the function of binding to DNA $\alpha$ subunits that partner with core binding factor $\beta$ [15] to control and guide the processes of cell differentiation, cell proliferation, and pedigree commitment in tissues [16], including the heart and vascular tissue $[17,18]$. Runx1 also regulates the development and transcription during hematopoiesis [19-21]. Recently, Runxl was demonstrated to be involved in the processes underlying adverse cardiac remodeling [22], and Runx1 activation in cardiomyocytes after $\mathrm{MI}$ is harmful to ventricular function [23]. However, to date, the protective role of Runx1 against adverse cardiac remodeling after $\mathrm{MI}$ in response to dihydrolycorine treatment remains unknown.

In this study, we explored the protective effects of dihydrolycorine in MI and elucidated the underlying mechanisms. Our data suggest that reducing the expression of Runx1 using dihydrolycorine treatment could rescue the adverse cardiac remodeling that occurs following MI, ultimately preventing the left ventricular dysfunction.

\section{Materials and Methods}

2.1. Ethics Statement and Surgical Ligation Model of MI. All experiments and procedures involving animals in this study were performed with humanitarian care in accordance with the Institutes of the First Affiliated Hospital of Wenzhou Medical University Health Guidelines for Laboratory Animals.

Male SD rats (10 weeks old) were anesthetized using $2.5 \%$ isoflurane and were subjected to thoracotomy $5 \mathrm{~mm}$ beneath the left atrial appendage to establish a MI model. The left anterior descending coronary artery was ligated using a 9-0 suture, and the success of the surgery was confirmed by the appearance of myocardial branching. The sham control rats did not undergo left coronary artery ligation. Before closing the chest, adenovirus constructs Ad-AE, Ad-Runx1, or Ad-sh-Runx1 green fluorescent protein (GFP) were injected into the left ventricular free wall of rats in the infarcted area ( $10 \mu \mathrm{L}$ for each of the four sites) using a $50 \mu \mathrm{L}$ needle (705RN, USA) [24-27]. After 1-week post-MI (baseline), rats were randomly assigned to groups that received treatment with or without saline, $5 \mathrm{mg} / \mathrm{kg}$ dihydrolycorine, $10 \mathrm{mg} / \mathrm{kg}$ dihydrolycorine, and $20 \mathrm{mg} / \mathrm{kg}$ dihydrolycorine for a month (Figure 1).
2.2. Echocardiography. Transthoracic echocardiography was performed using a Philips iE33 system (Philips Medical, Netherlands), and left ventricular end-systolic diameter (LVESD) and left ventricular end-diastolic diameter (LVEDD) were recorded. Next, left ventricular fraction shortening (LVFS) and left ventricular ejection fraction (LVEF) were calculated using a computer algorithm after three successive cardiac cycles.

2.3. RNA-seq Based on Gene Expression Data Analysis. MI RNA-seq data based on the GSE114695 [28] dataset was acquired from the Gene Expression Omnibus (GEO) database (https://www.ncbi.nlm.nih.gov/geo/). Differentially expressed MI-related genes were screened using Microsoft Excel with the thresholds of $|\log 2 \mathrm{FC}|>2.0$ and $P$ value after correction (adj.P.Val) $<0.05$. A heat map of differentially expressed MI-related genes was constructed using GraphPad Prism 7.0.

Interacting proteins specific for the Runxl gene were searched in the GeneCards (https://www.genecards.org/) and String databases (https://string-db.org/).

2.4. Molecular Docking. Molecular docking studies were performed to determine the binding mode between dihydrolycorine and human Runx1 using AutoDock Vina 1.1.2 (The Scripps Research Institute, San Diego, USA).

2.5. Primary Culture of Neonatal Cardiomyocytes and Cell Culture. Primary cardiomyocytes were isolated from the left ventricle of 1-day-old SD rats according to a previously described protocol [24]. Primary cardiomyocytes were cultured in a $5 \% \mathrm{CO}_{2}, 21 \% \mathrm{O}_{2}$, and balanced nitrogen incubator at $37^{\circ} \mathrm{C}$ under normoxic conditions. Other primary cardiomyocytes were cultured in $1 \% \mathrm{O}_{2}, 5 \% \mathrm{CO}_{2}$, and balanced nitrogen at $37^{\circ} \mathrm{C}$ (oxygen-glucose deprivation) $[5,29]$ to establish a MI model in vitro (hypoxic condition). Hypoxia cardiomyocytes were incubated with 1.0, 5.0, 10, and $15 \mu \mathrm{M}$ dihydrolycorine for $24 \mathrm{~h}$. Experimental cardiomyocytes were transfected with adenovirus (Ad-Runx1, or Adsh-Runx1; $10 \mathrm{~mL} / \mathrm{mL}$, MOI: $100: 1)$ in serum-free DMEM for $6-8 \mathrm{~h}$ and treated under hypoxic or normoxic conditions, with or without dihydrolycorine for an additional $24 \mathrm{~h}$.

2.6. Immunofluorescence Staining. Experimental primary cardiomyocytes in 48-orifice-plates were fixed using $4 \%$ paraformaldehyde, permeabilized using $0.5 \%$ Triton X-100, blocked using $4 \%$ goat serum, and then incubated with primary antibodies against SM $\alpha$-actin ( $\alpha$-SMA, ab5694), and connexin $43(\mathrm{ab} 11370)$ at $4^{\circ} \mathrm{C}$ overnight. After incubation 


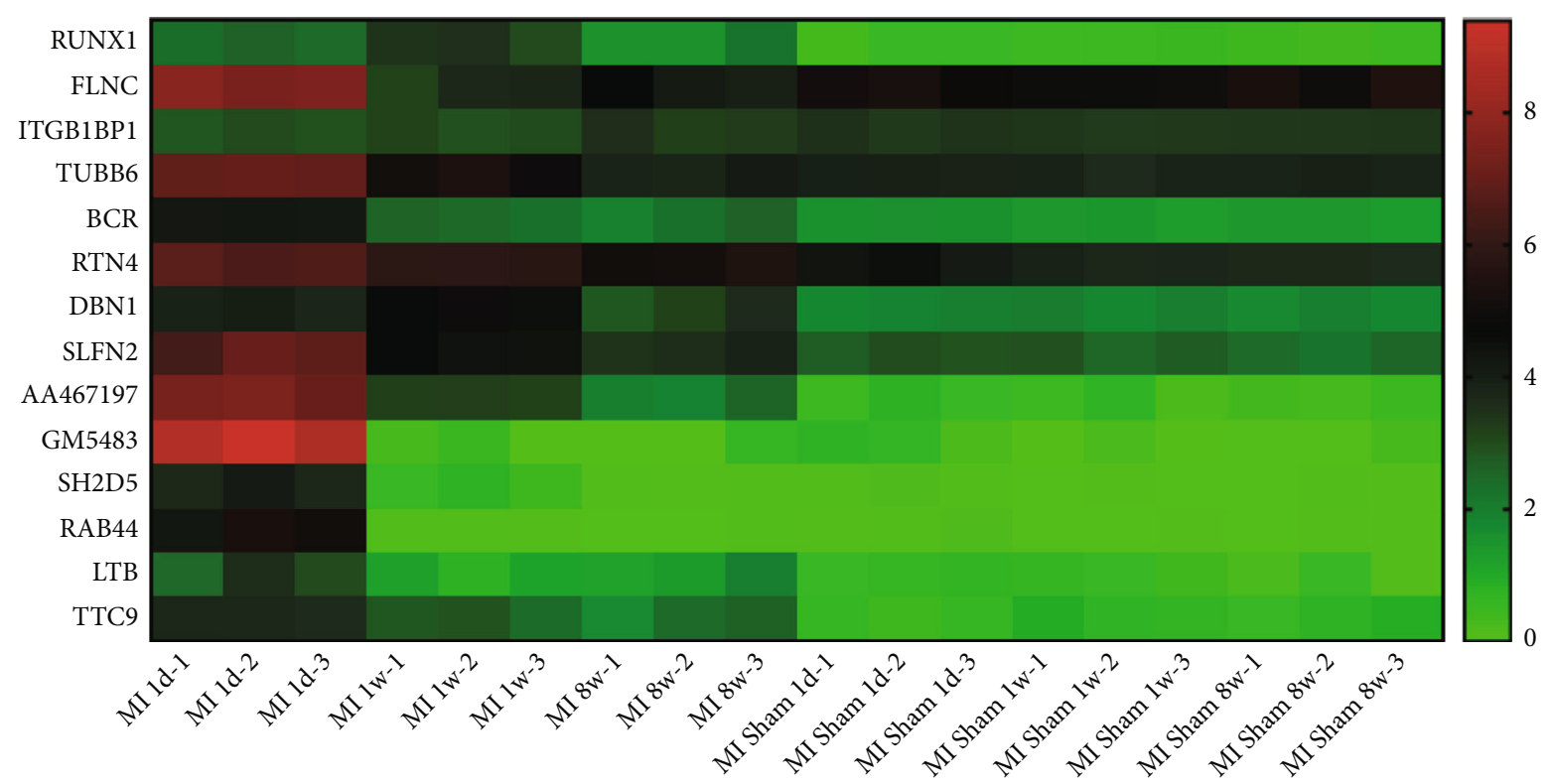

(a)
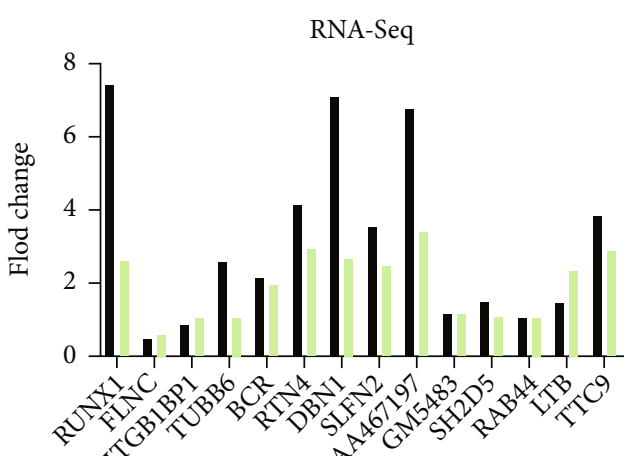

MI $1 \mathrm{~W}$

MI 8W

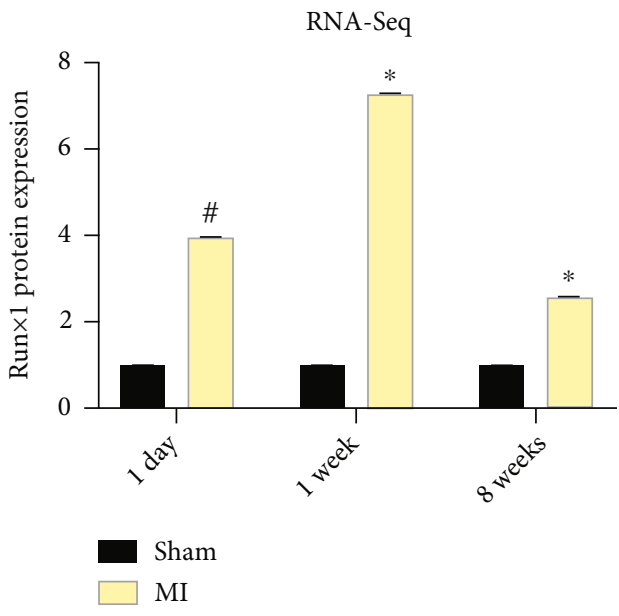

(b)

(c)
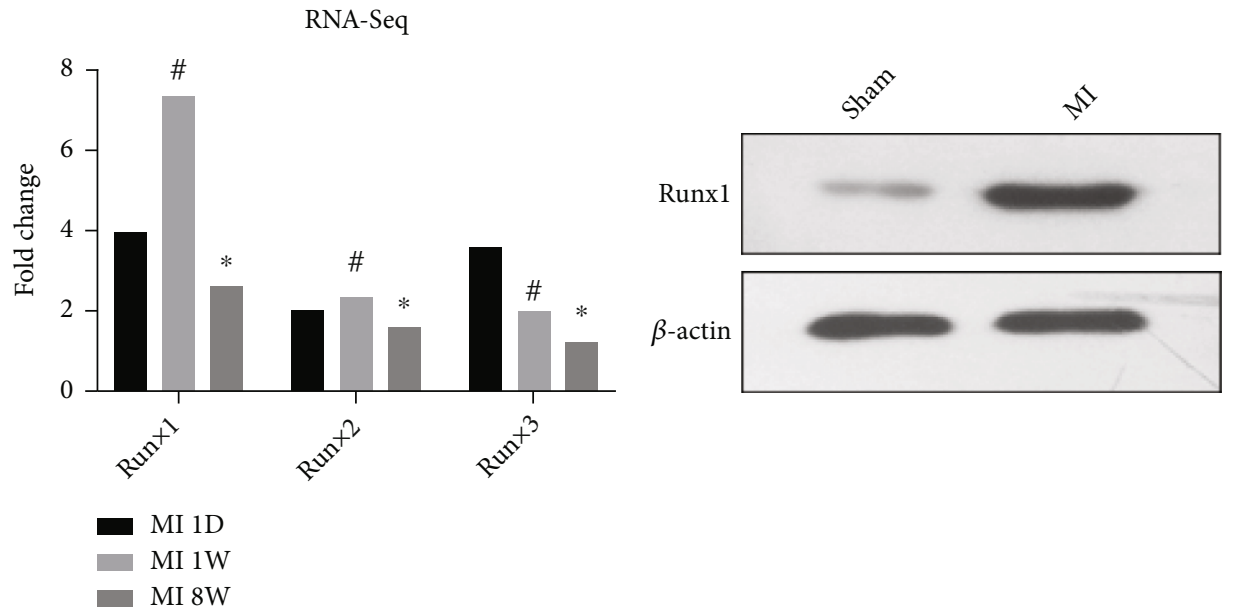

(d)

(e)

FIgURe 2: Continued. 


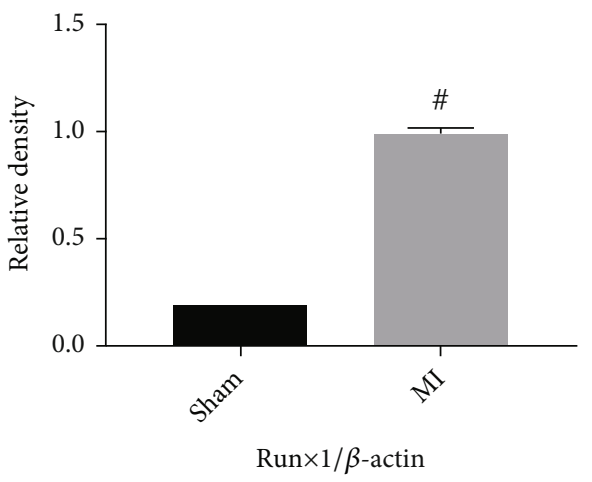

(f)

Figure 2: Runx1 was the most differentially expressed myocardial infarction- (MI-) related gene. (a) The heat map of the top 14 differentially expressed MI-related genes in the RNA-sequencing dataset GSE1146395. (b) Comparison of mRNA expression levels of the MI-related genes obtained from RNA-sequencing data (GSE1146395). (c) Expression of Runx1 mRNA as determined by RNA-sequencing data. (d) Comparison of the RUNX (runt-related) family mRNA levels from RNA-sequencing data. (e, f) Relative protein expression of Runx1 according to western blotting. ${ }^{\#} P<0.05$ vs. sham and ${ }^{*} P<0.05$ vs. MI; experiments were repeated more than three times.

TABLE 1: Dihydrolycorine improved the cardiac function in the hearts of MI rats.

\begin{tabular}{|c|c|c|c|c|c|}
\hline \multirow{2}{*}{ Group $(n=8)$} & \multirow{2}{*}{ Sham } & \multirow{2}{*}{ MI+saline } & \multicolumn{3}{|c|}{ MI+dihydrolycorine } \\
\hline & & & $5 \mathrm{mg} / \mathrm{kg}$ & $10 \mathrm{mg} / \mathrm{kg}$ & $20 \mathrm{mg} / \mathrm{kg}$ \\
\hline LVEF (\%) & $79.39 \pm 1.21$ & $50.32 \pm 3.53$ & $53.13 \pm 2.03^{* \#}$ & $57.36 \pm 1.29^{* \#}$ & $64.23 \pm 3.62^{* \#}$ \\
\hline LVFS\% & $39.1 \pm 1.3$ & $21.2 \pm 3.5$ & $24.3 \pm 4.1^{*}$ & $28.1 \pm 2.6^{* \#}$ & $31.3 \pm 2.4^{* \#}$ \\
\hline LVESD (mm) & $2.4 \pm 0.31$ & $3.6 \pm 0.42$ & $3.4 \pm 0.23^{* \#}$ & $3.2 \pm 0.34^{* \#}$ & $2.9 \pm 0.23^{* \#}$ \\
\hline LVEDD (mm) & $3.7 \pm 0.3$ & $4.9 \pm 0.6$ & $4.6 \pm 0.3^{* \#}$ & $4.3 \pm 0.4^{\#}$ & $4.0 \pm 0.2^{* \#}$ \\
\hline $\mathrm{E} / \mathrm{A}$ ratio & $2.13 \pm 0.56$ & $1.48 \pm 0.36$ & $1.62 \pm 0.41^{* \#}$ & $1.73 \pm 0.32^{* \#}$ & $1.83 \pm 0.36^{* \#}$ \\
\hline HR (bmp) & $520 \pm 8$ & $480 \pm 5$ & $487 \pm 2^{*}$ & $492 \pm 4^{* \#}$ & $501 \pm 7^{* \#}$ \\
\hline
\end{tabular}

Values are mean $\pm \mathrm{SD}, P<0.05$. The data are presented as means and $\mathrm{SD} .{ }^{*} P<0.05$ versus sham; ${ }^{*} P<0.05$ versus MI + saline. LVEDD: left ventricular end-diastolic dimension; LVESD: left ventricular end-systolic dimension; LVEF: left ventricular ejection fraction; LVFS: left ventricular systolic function; HR: heart rate.

with DyLight 488 and 594 AffiniPure Goat IgG $(\mathrm{H}+\mathrm{L})$ for $1 \mathrm{~h}$ at $37^{\circ} \mathrm{C}$, the cardiomyocytes were counterstained with $0.1 \mu \mathrm{g} / \mathrm{mL} 4^{\prime}$,6-diamidino-2-phenylindole (DAPI) (P36941; Invitrogen) for $3 \mathrm{~min}$ and images were obtained using a Nikon Eclipse Ti-U fluorescence microscope $(\times 400$ or $\times 200)$.

\subsection{Wheat Germ Agglutinin (WGA) Staining. WGA staining} (iFluor $^{\mathrm{TM}}$ 594-Wheat Germ Agglutinin Conjugate, AAT Bioquest) was performed to determine the size of cardiomyocytes and to find out whether cardiomyocytes were hypertrophic. Left ventricular tissues from infarcted areas in rat hearts were embedded in paraffin and cut into $5 \mu \mathrm{m}$ thick sections, which were then incubated with $5 \mu \mathrm{g} / \mathrm{mL}$ WGA (L4895; Sigma-Aldrich) in the dark at room temperature and then stained with DAPI for $3 \mathrm{~min}$. Images were obtained using a Nikon Eclipse Ti-U fluorescence microscope (Minato-ku, Tokyo, Japan) and analyzed using Image J software (National Institutes of Health).

2.8. Western Blotting. Total protein samples were obtained from left ventricular tissues from infarcted areas in rat's hearts, cardiomyocytes, and H9C2 cells (ATCC, USA). A bicinchoninic acid reagent was used according to a previously described protocol to ensure identical protein concentrations in each protein sample [30]. Protein samples were subjected to sodium dodecyl sulfate-polyacrylamide gel electrophoresis (SDS-PAGE) and then transferred onto $0.45 \mu \mathrm{m}$ polyvinylidene difluoride membranes (PVDF) (Millipore, Billerica, MA, USA). The membranes were blocked using 5\% milk in TBST at room temperature for $1 \mathrm{~h}$ and then incubated with primary antibodies, including Runx1 (ab92336; Abcam), TGF $\beta$ (ab215715), p-smad3 (ab52903), smad3 (ab40854), collagen I (ab34710), Bax (ab32503), Bcl-2 (ab32124), CBFB (ab133600), CEBPA (ab40761), and $\beta$-actin (ab8226) overnight at $4^{\circ} \mathrm{C}$. PVDF membranes were incubated with horseradish peroxidase-conjugated goat anti-mouse and anti-rabbit IgG $(\mathrm{H}+\mathrm{L})(1: 5000$; Abbkine, Redlands, CA) on the following day and visualized using an ECL chemiluminescent kit (Sigma, United States). Quantity One 5.0 software (Bio-Rad Laboratories, Inc., Hercules, CA, USA) was used for quantification.

2.9. Pull-Down Assay. Binding buffer was added to the mixed lysate obtained from primary cardiomyocytes containing two proteins that predicted interaction, and the sample was 

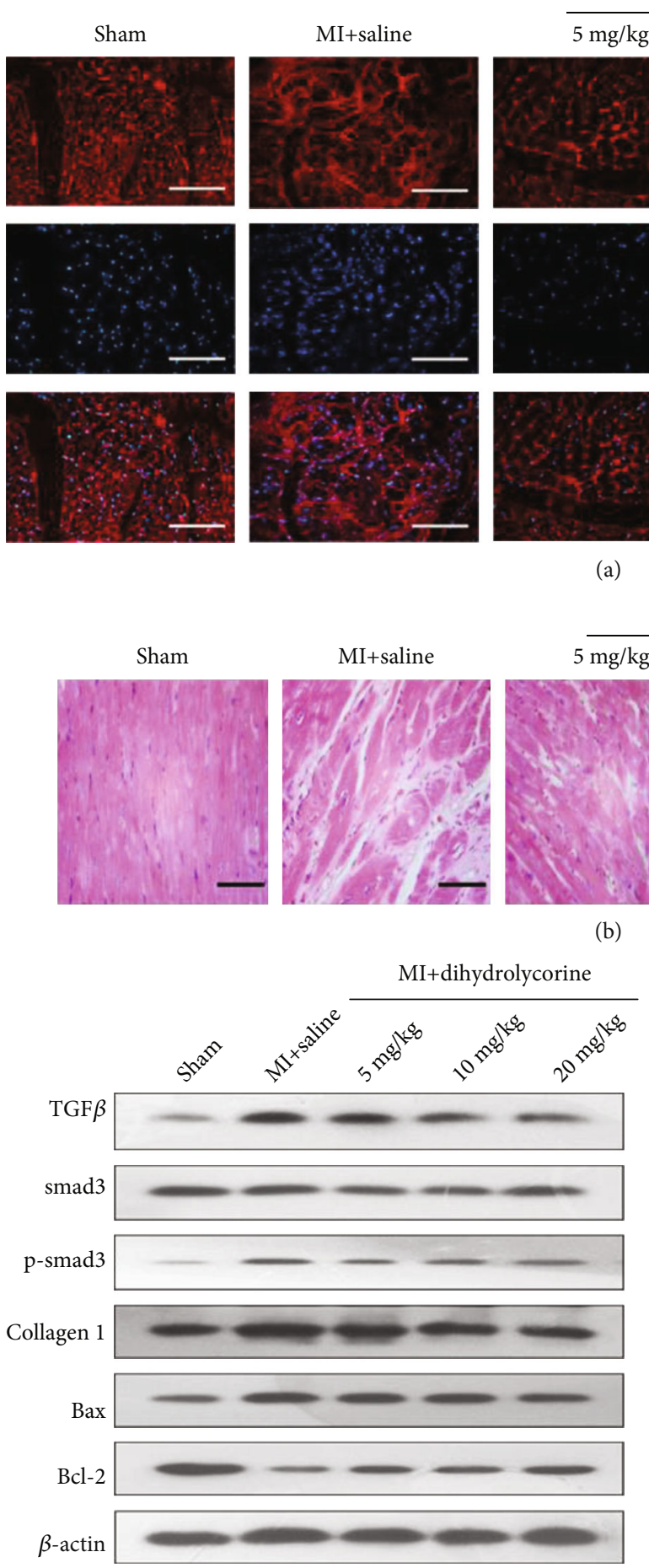

(c)

(a)

b)
MI+dihydrolycorine
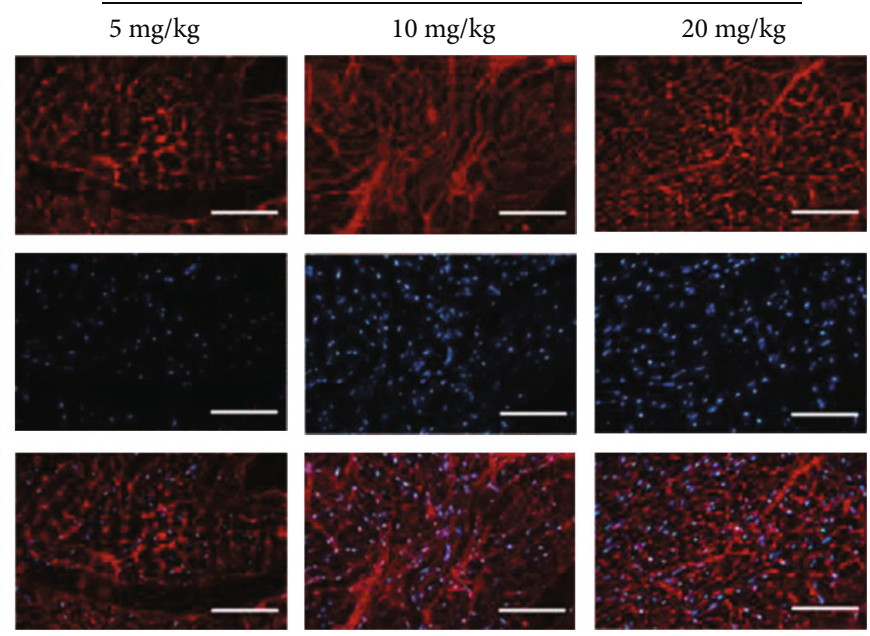

MI+dihydrolycorine
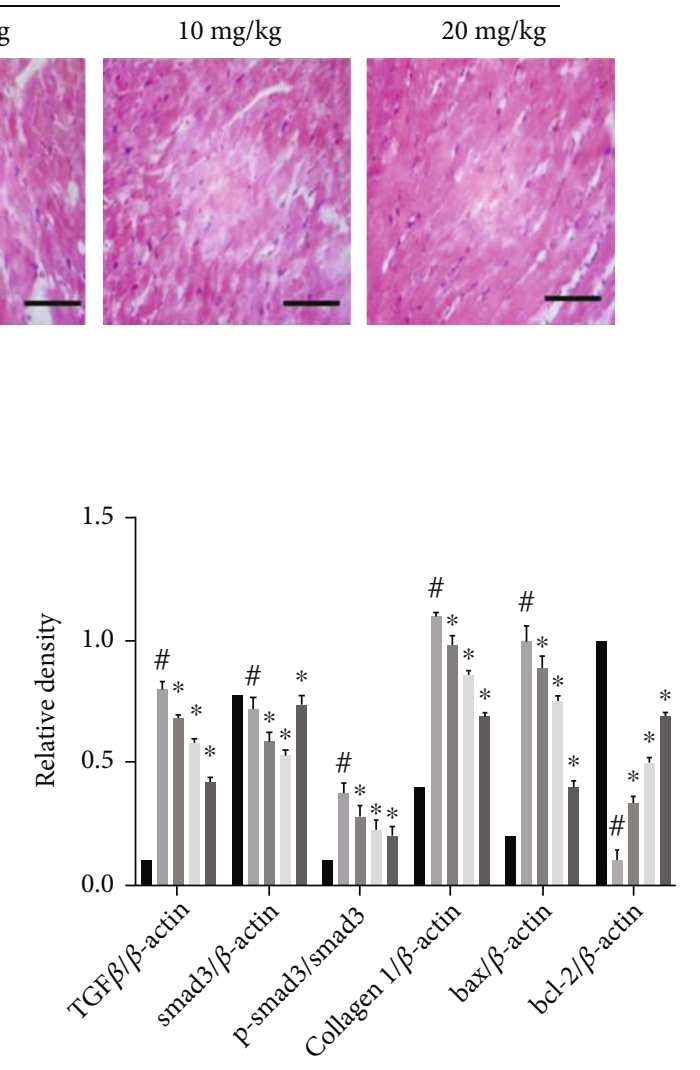

(d)

Figure 3: Continued. 


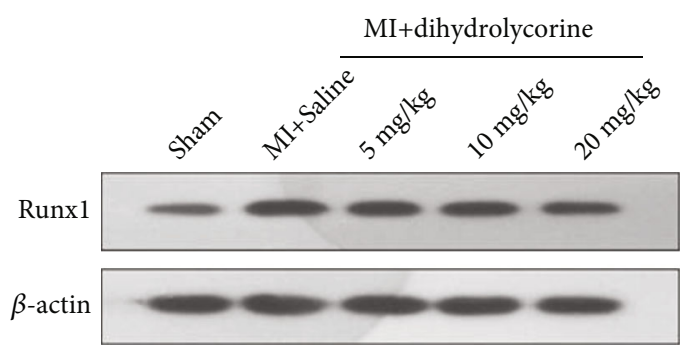

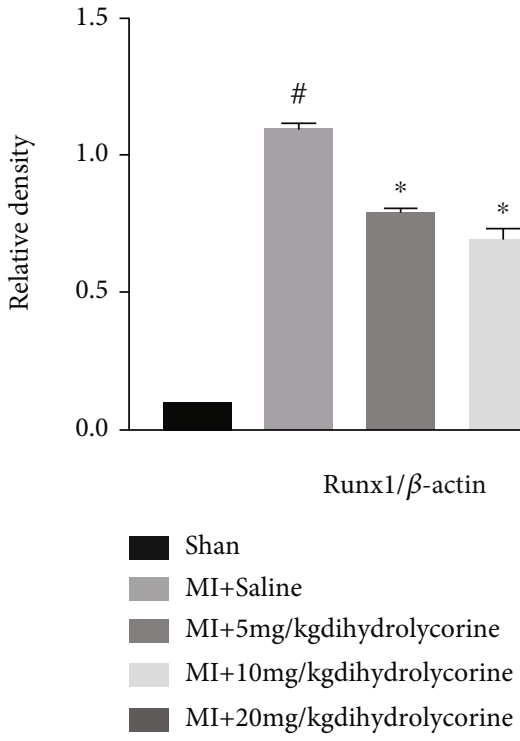

(f)

Figure 3: Dihydrolycorine reduced the fibrosis of the myocardium after myocardial infarction (MI). (a) Cardiac myocyte hypertrophy was determined by using wheat germ agglutinin (WGA, magnification $\times 100)$ staining of left ventricular tissue from rats. Scale bar $=100 \mu \mathrm{m}$. $(\mathrm{b})$ Myocardial fibers in myocardial tissue sections were stained with hematoxylin and eosin (H\&E, magnification $\times 40)$. Scale bar $=200 \mu \mathrm{m}$. (c, d) Relative protein expression of collagen I, TGF $\beta, \operatorname{smad} 3, \mathrm{p}-\mathrm{smad} 3, \mathrm{Bax}$, and Bcl-2 according to western blotting. (e, f) Relative protein expression with representative gel blots of Runx1 by western blot analysis. ${ }^{\#} P<0.05$ vs. sham and ${ }^{*} P<0.05$ vs. MI; experiments were repeated more than three times.

then rotated for bonding determination at $4^{\circ} \mathrm{C}$ for $2-4 \mathrm{~h}$. After centrifugation for $2 \mathrm{~min}$ at $4^{\circ} \mathrm{C}$, the supernatant was discarded, and the cells were washed with $1 \mathrm{~mL}$ binding buffer 5 to 6 times. Loading buffer was added to the 20-30 $\mu \mathrm{L}$ liquid that remained after the last wash. Western blotting analysis was performed.

2.10. Hematoxylin and Eosin (HÆE) Staining. H\&E staining was used to assess the degree of myocardial fibrosis and evaluate the general morphology of the myocardium. Left ventricular tissues from infarcted areas in rat hearts were embedded in paraffin and cut into $5 \mu \mathrm{m}$ thick sections, which were then incubated with hematoxylin and eosin (H\&E). Images were obtained using a Nikon Eclipse Ti-U fluorescence microscope (Minato-ku, Tokyo, Japan) and analyzed using Image J software (National Institutes of Health).

2.11. Statistical Analyses. All experiments were repeated a minimum of three times. Data are presented as the mean \pm standard deviation. Two-tailed paired or unpaired Student's $t$-tests were performed to compare two normally distributed groups with normal data distribution; one-way analysis of variance (ANOVA) or two-way ANOVA followed by Bonferroni's correction was used for multiple comparisons. If the normality of the data could not be confirmed, MannWhitney tests were used. All data were analyzed using SPSS version 26.0 software (SPSS Inc. USA, IL). Statistical significance was set at $P<0.05$.

\section{Results}

3.1. MI-Related Genes Were Differentially Expressed, and Runx1 Was Highly Expressed after MI. The differentially expressed MI-related gene profiles of the infarct area were screened from the GSE1146395 dataset obtained from the GEO database based on a threshold of $|\log 2 \mathrm{FC}|>2.0$, and adj.P.Val $<0.05$. The top 14 differentially expressed MIrelated genes at day 1 , week 1 , and eight weeks after MI surgery are shown in the heat map (Figure 2(a)), with three mice in each period. After a data analysis of the RNA-sequence, we determined that Runx1 was the most differentially expressed MI-related gene (Figure 2(b)). Figure 2(c) indicates that the highest increase in the mRNA levels of Runxl occurred at week 1 after MI surgery compared to the levels in sham rats. In the Runt-related family (Runx1, Runx2, and Runx3), Runx1 expression was most significantly elevated (Figure 2(d)) according to the RNA-sequence analysis. We confirmed these results using western blotting and found that Runx1 was upregulated in the infarct area 1 week after MI surgery (Figures 2(e) and 2(f)).

3.2. Dihydrolycorine Improved Cardiac Function of MI Rats and Downregulated Runx1. To explore the cardioprotective function of dihydrolycorine at week 1 after MI surgery and at 12 weeks after 5,10 , and $20 \mathrm{mg} / \mathrm{kg}$ dihydrolycorine treatments, we determined the cardiac function of rats using transthoracic echocardiography. As shown in Table 1, as the concentration of dihydrolycorine increased, cardiac 


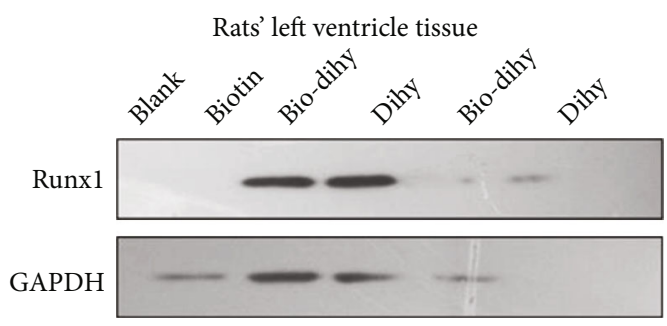

(a)

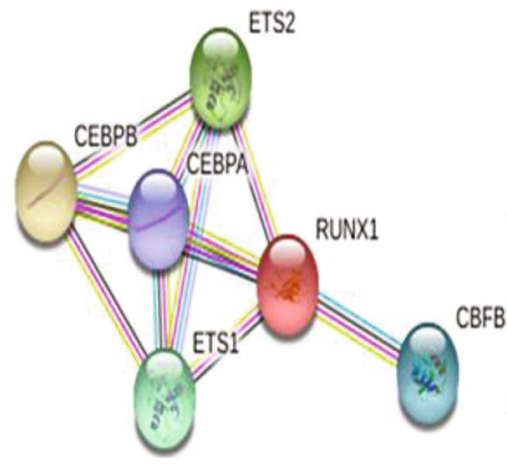

(c)

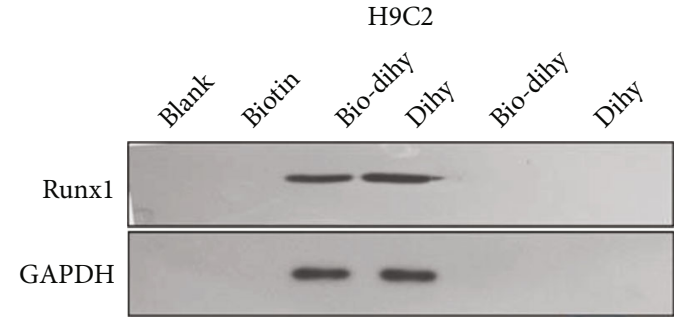

(b)

Cardiomyocyte

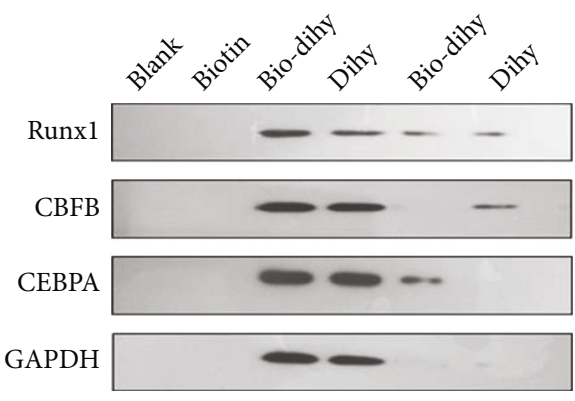

(d)

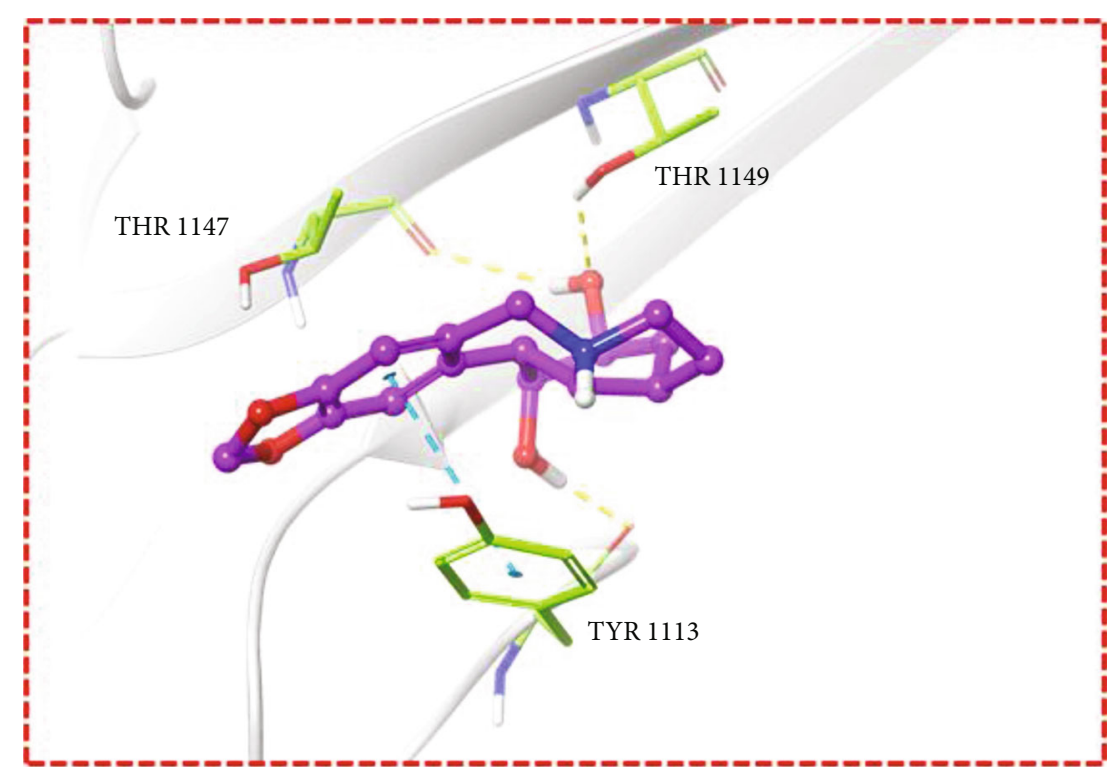

(e)

Figure 4: Continued. 


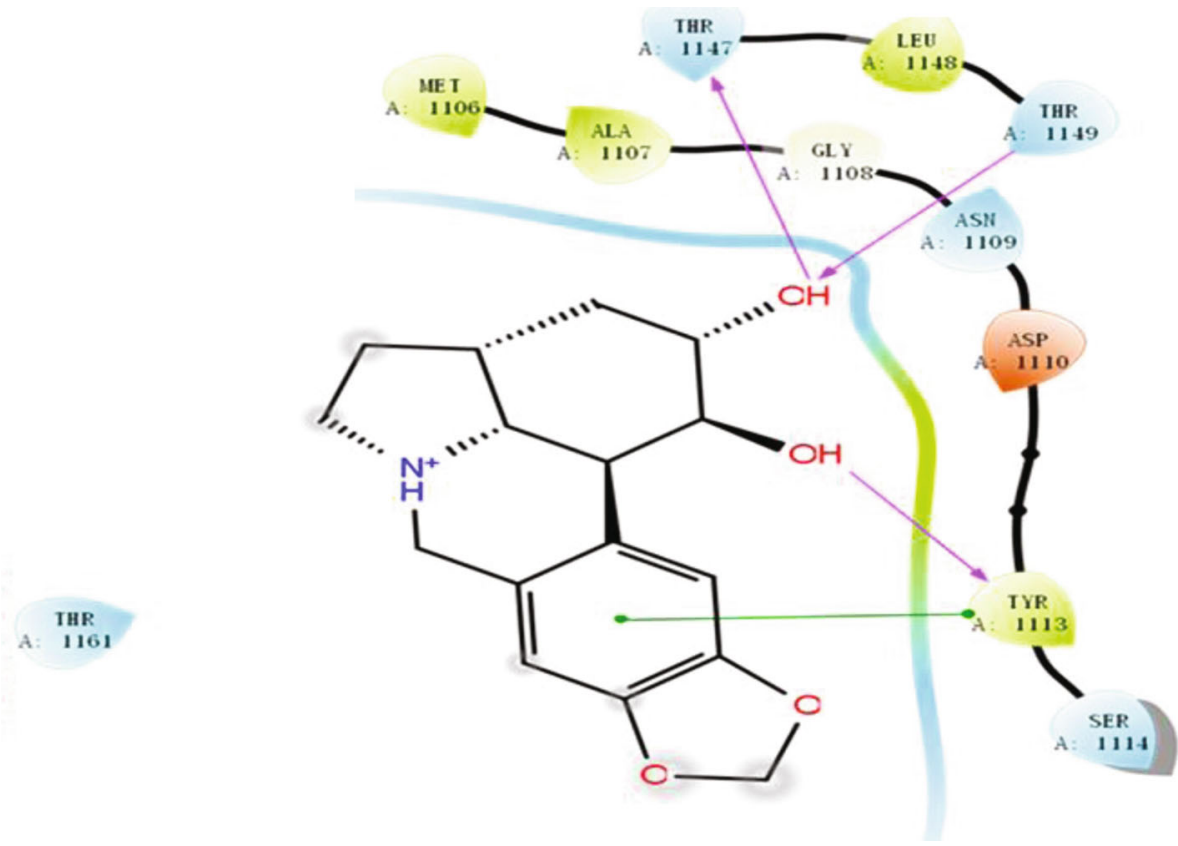

(f)

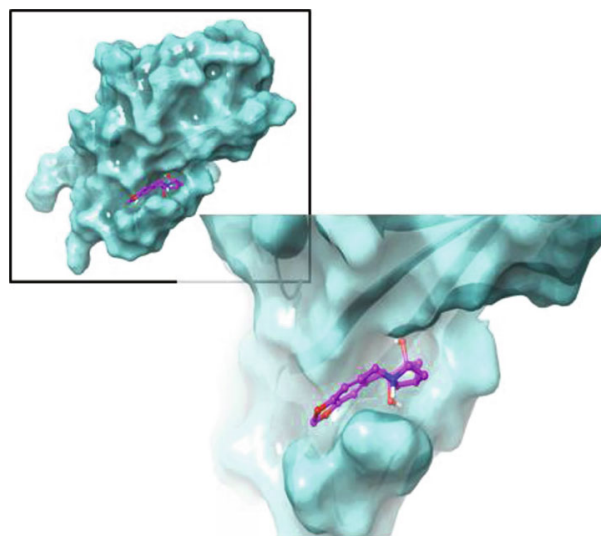

(g)

Figure 4: Identification of dihydrolycorine binding proteins. (a) Protein expression with representative gel blots of Runx1 according to western blotting. Lysates prepared from (a) rats' ventricle tissues and (b) H9C2 cells were added to the streptavidin-agarose beads with Bio-Dihy that were previously added to the streptavidin-agarose beads with prior incubation. (c) The upstream signaling proteins of Runx1 according to GeneCards and the STRING Interaction Network. (d) Pull-down assay was used to assess dihydrolycorine binding to Runx1, CBFB, and CEBPA. Lysates were obtained from primary cardiomyocytes. (e-g) Representative images were obtained by molecular docking between dihydrolycorine and Runxl.

dysfunction was further suppressed, which was indicated by increased LVEF, LVFS, and E/A ratio and decreased LVESD and LVEDD (Table 1). As expected, the left ventricular cardiomyocytes subjected to WGA staining revealed that the MI-associated enlargement of cardiomyocytes could be mitigated by treatment with a $20 \mathrm{mg} / \mathrm{kg}$ dihydrolycorine (Figure 3(a)). Further analysis revealed that dihydrolycorine can alleviate the occurrence of irregular myocardial fibers and obscure intercellular borders in the infarcted area, which showed the initiation of myocardial necrosis, as evidenced by $H \& E$ staining (Figure 3(b)). Therefore, in all the subsequent animal experiments, dihydrolycorine was administered at a concentration of $20 \mathrm{mg} / \mathrm{kg}$. Furthermore, we observed the downregulation of fibrotic genes, including col- lagen $\mathrm{I}, \mathrm{TGF} \beta$, and $\mathrm{p}$-smad3, in the MI rats treated with dihydrolycorine (Figures 3(c) and 3(d)), downregulation of the apoptosis gene Bax, and upregulation of the apoptosis gene Bcl-2. Most importantly, upregulation of Runx1 after MI surgery was inhibited by dihydrolycorine treatment (Figures 3(e) and 3(f)). These results confirmed that in vitro dihydrolycorine treatment could prevent MI-induced myocardial fibrosis and cardiomyocyte hypertrophy and may thus mediate Runx1 to exert a cardioprotective effect.

\subsection{Dihydrolycorine May Directly Inhibit Runx1 and Enhance} Its Activity. To determine whether dihydrolycorine binds to Runx1 in cardiomyocytes, we performed biotinylated protein interaction pull-down assays. Biodihydrolycorine (Dihy) was 


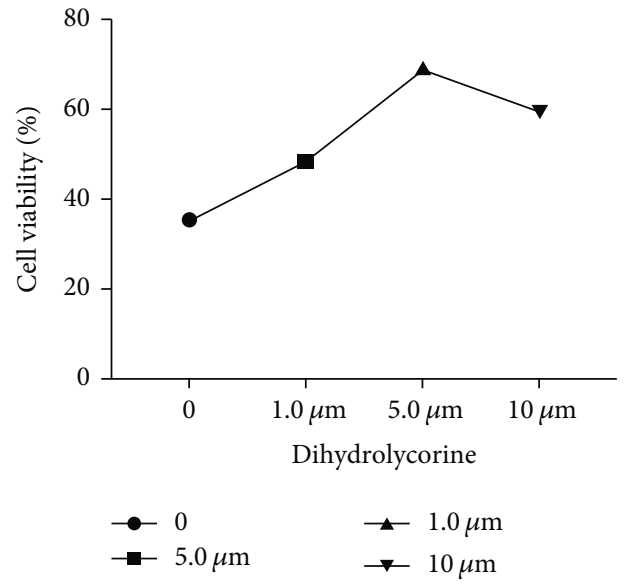

(a)

(c)

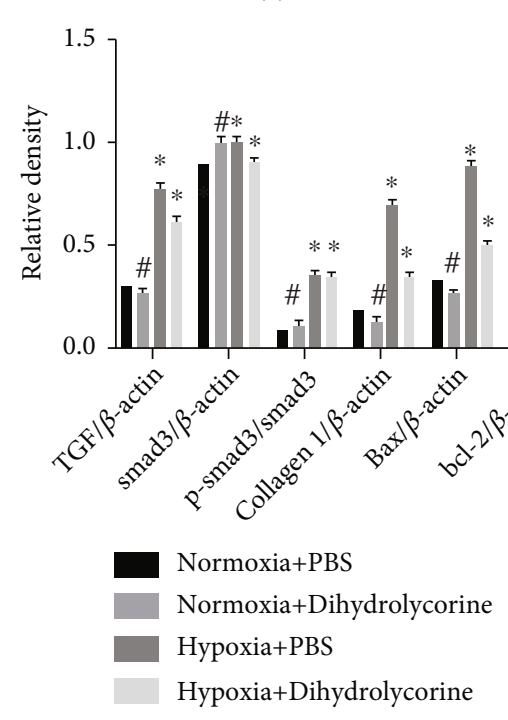

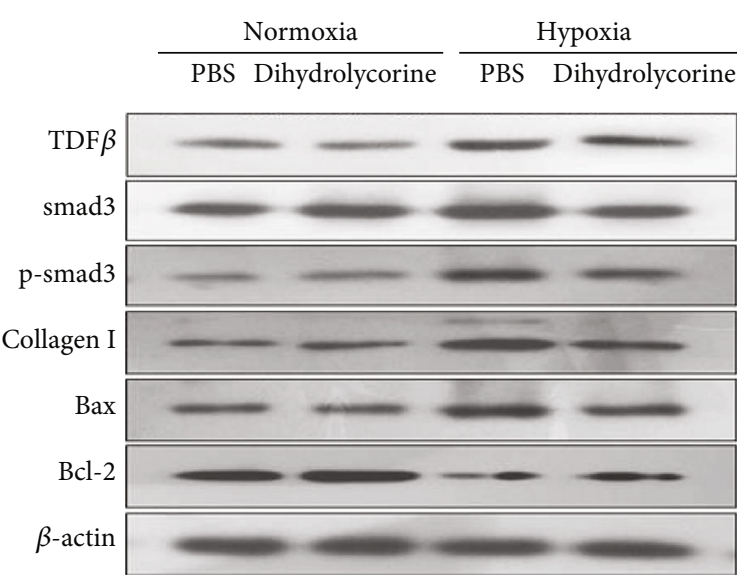

(b) (d)

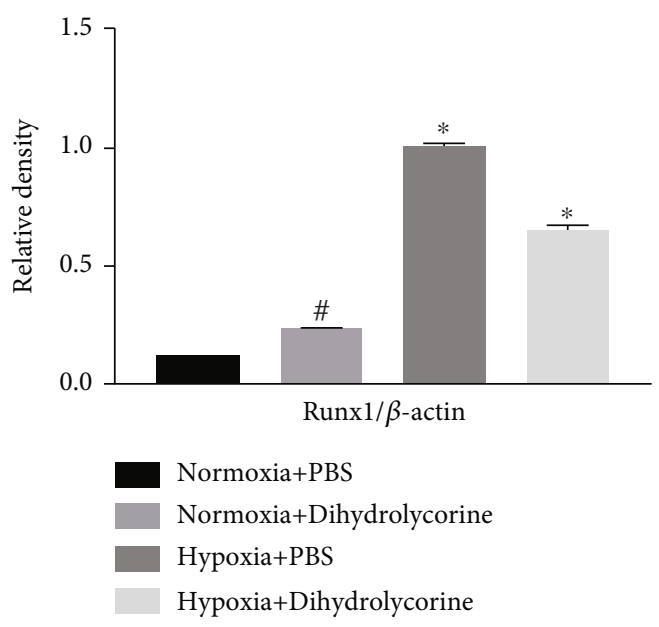

(e)

Figure 5: Continued. 


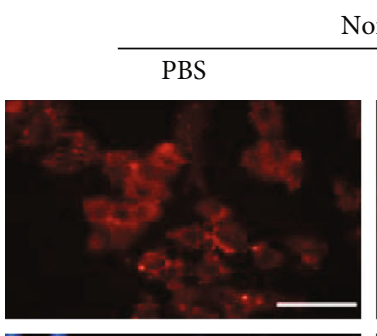

Normoxia Dihydrolycorine
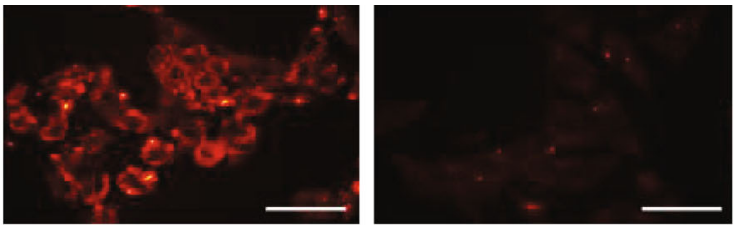
Hypoxia
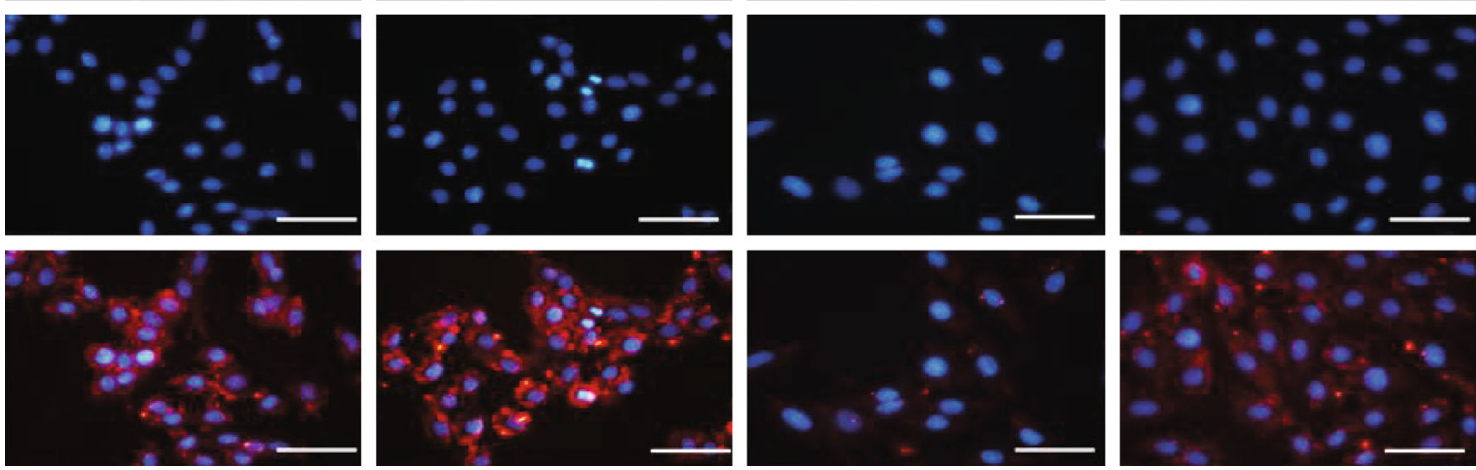

(f)

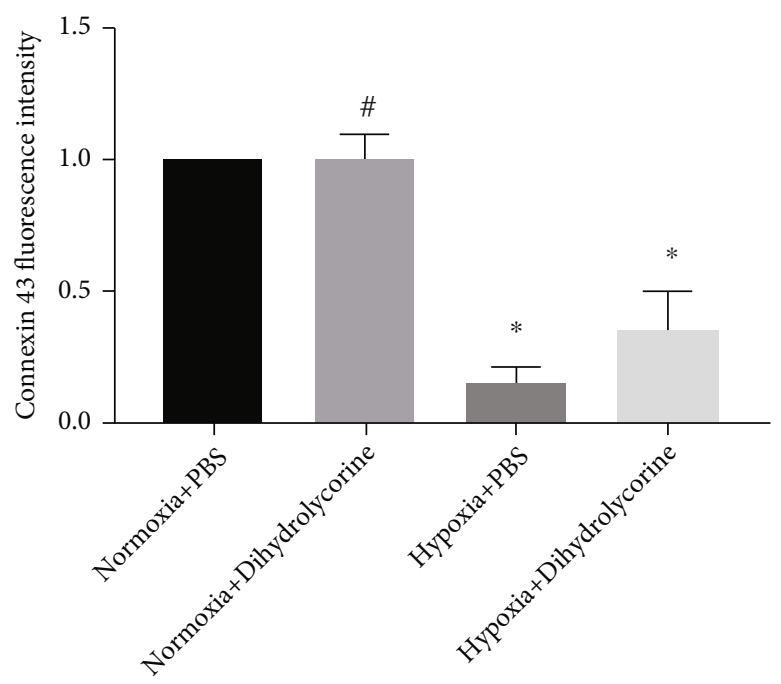

(g)

Figure 5: Continued. 


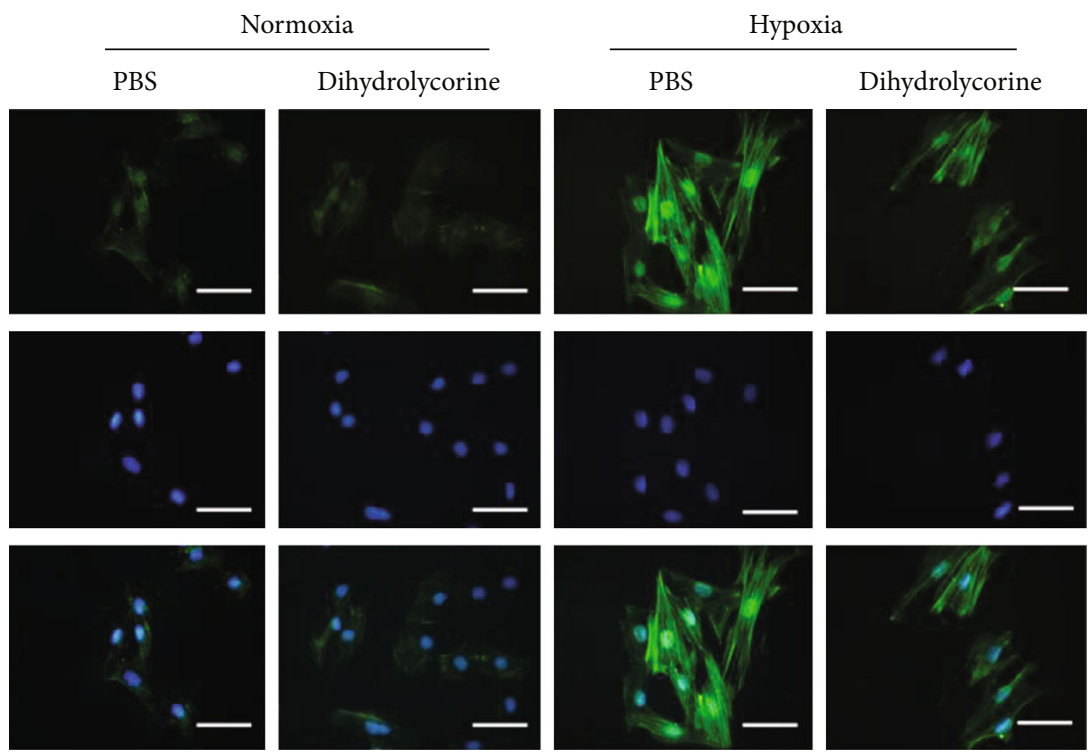

(h)

FIGURE 5: Dihydrolycorine rescued fibrosis and apoptosis in hypoxia-treated cardiomyocytes and induced decreased Runx1 expression. (a) Hypoxia-treated cardiomyocytes were treated with $1.0,5.0$, or $10 \mu \mathrm{M}$ dihydrolycorine for $24 \mathrm{~h}$, followed by DNA quantification to measure cell number/cell proliferation. (b, c) Relative protein expression of collagen I, TGF $\beta$, smad3, p-smad3, Bax, and Bcl-2 according to western blotting. (d, e) Relative protein expression of Runx1 according to western blotting. (f) Immunofluorescence staining of connexin 43 (red, magnification $\times 400$ ). Scale bar $=50 \mu \mathrm{m}$. (g) Bar graphs of fluorescence intensity of connexin 43. (h) Immunofluorescence staining of $\alpha$-SMA (green, magnification $\times 400$ ). Scale bar $=50 \mu \mathrm{m}$. ${ }^{\#} P<0.05$ vs. normoxia and ${ }^{*} P<0.05$ vs. hypoxia; experiments were repeated more than three times.

incubated with streptavidin-agarose beads, and lysates from the left ventricle tissues of rats and $\mathrm{H} 9 \mathrm{C} 2$ cells were added. Biodihydrolycorine binds to Runxl protein in lysates from both rats' left ventricle tissue and H9C2 cells (Figures 4(a) and 4(b)). Furthermore, when we explored upstream signaling proteins of Runx1 (CBFB and CEBPA), which were screened by GeneCards and the STRING Interaction Network (Figure 4(c)), we found that these proteins did not interact with dihydrolycorine according to the results of the pulldown assay (Figure 4(d)). These results demonstrate that dihydrolycorine directly binds to Runx1 rather than to its upstream mediators.

To explore the interaction between Runx1 and dihydrolycorine, we performed molecular docking using AutoDock Vina 1.1.2. and determined the crystal structure of Runx1 (PDB ID:1LJM) using a simulation study. Figure 4(e) shows three binding sites on Runx1, including THR 1147, THR 1149, and TYR 1113, which form three hydrogen bond interactions and one Pi-Pi interaction with TYR 1113, with an affinity of $-6.1 \mathrm{kcal} / \mathrm{mol}$. The chemical structure of the binding site between dihydrolycorine and Runx1 is shown in Figure 4(f). Figure 4(g) shows the crystal structure of the binding site between dihydrolycorine and Runx1 in the three-dimensional diagram.

\subsection{Dihydrolycorine Exerted Positive Effects on Hypoxia-} Treated Cardiomyocytes. We confirmed that dihydrolycorine exhibited cardioprotective effects in vitro. First, we found that the number of hypoxia-treated cardiomyocytes was significantly higher following the culture with dihydrolycorine at concentrations of $1.0,5.0,10$, and $15 \mu \mathrm{M}$ for $24 \mathrm{~h}$ than that observed in the control group; however, these numbers began decreasing upon at treatment with $15 \mu \mathrm{M}$ dihydrolycorine (Figure 5(a)). For all subsequent cell experiments, dihydrolycorine was added at a concentration of $10 \mu \mathrm{M}$. Second, we observed that the expression levels of fibrotic genes and apoptosis genes, including collagen I, TGF $\beta, \mathrm{p}$-smad3, and Bax, were decreased in the dihydrolycorine-treated hypoxia-cardiomyocytes (Figures 5(b) and 5(c)). Third, Runx1 was also downregulated in dihydrolycorine-treated hypoxia-treated cardiomyocytes (Figures 5(d) and 5(e)). Fourth, we identified that the cardiac functional protein, punctuate gap junction protein connexin 43 was rescued by dihydrolycorine treatment in hypoxia-cardiomyocytes in proximity to the plasma membrane according to immunofluorescent staining results (Figures 5(f) and 5(g)). Finally, we investigated the overexpression of $\alpha$-SMA in hypoxia-cardiomyocytes that resulted in cardiomyocyte fibrosis as assessed by immunofluorescence staining and found that the levels of this protein were reduced after dihydrolycorine treatment (Figure 5(h)). Taken together, these results suggest that dihydrolycorine prevents hypoxiainduced cardiomyocyte fibrosis and regulates the expression of Runx1.

3.5. Dihydrolycorine Suppressed Myocardial Fibrosis after MI by Reducing Runx1 Levels In Vivo and In Vitro. We next investigated the specific mechanisms underlying the positive effect of dihydrolycorine in MI rats and hypoxia-treated cardiomyocytes, while confirming whether Runx1 plays an 


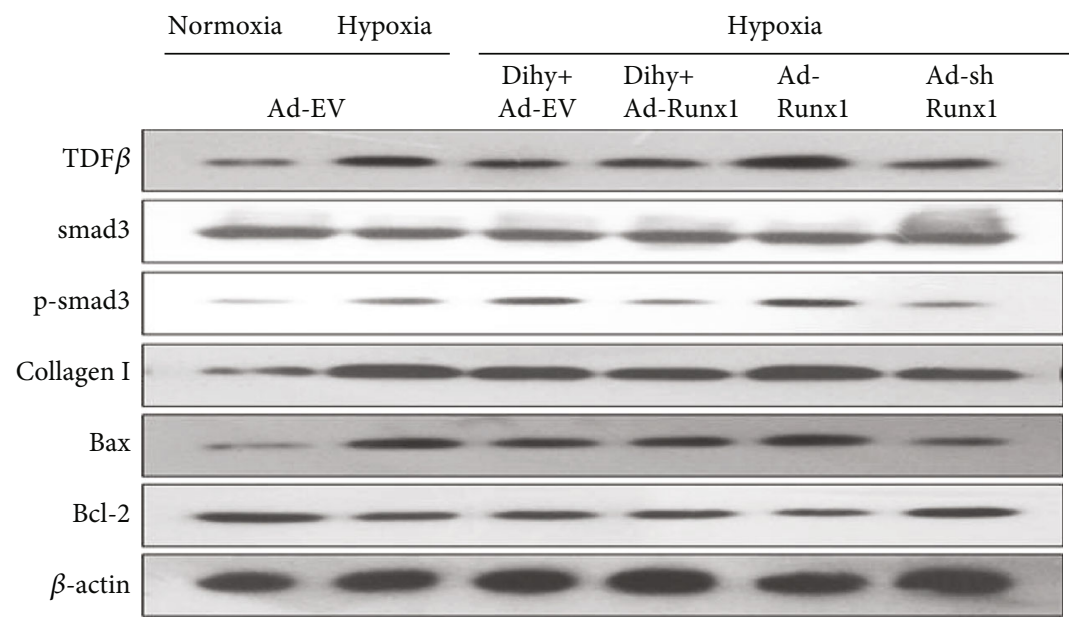

(a)
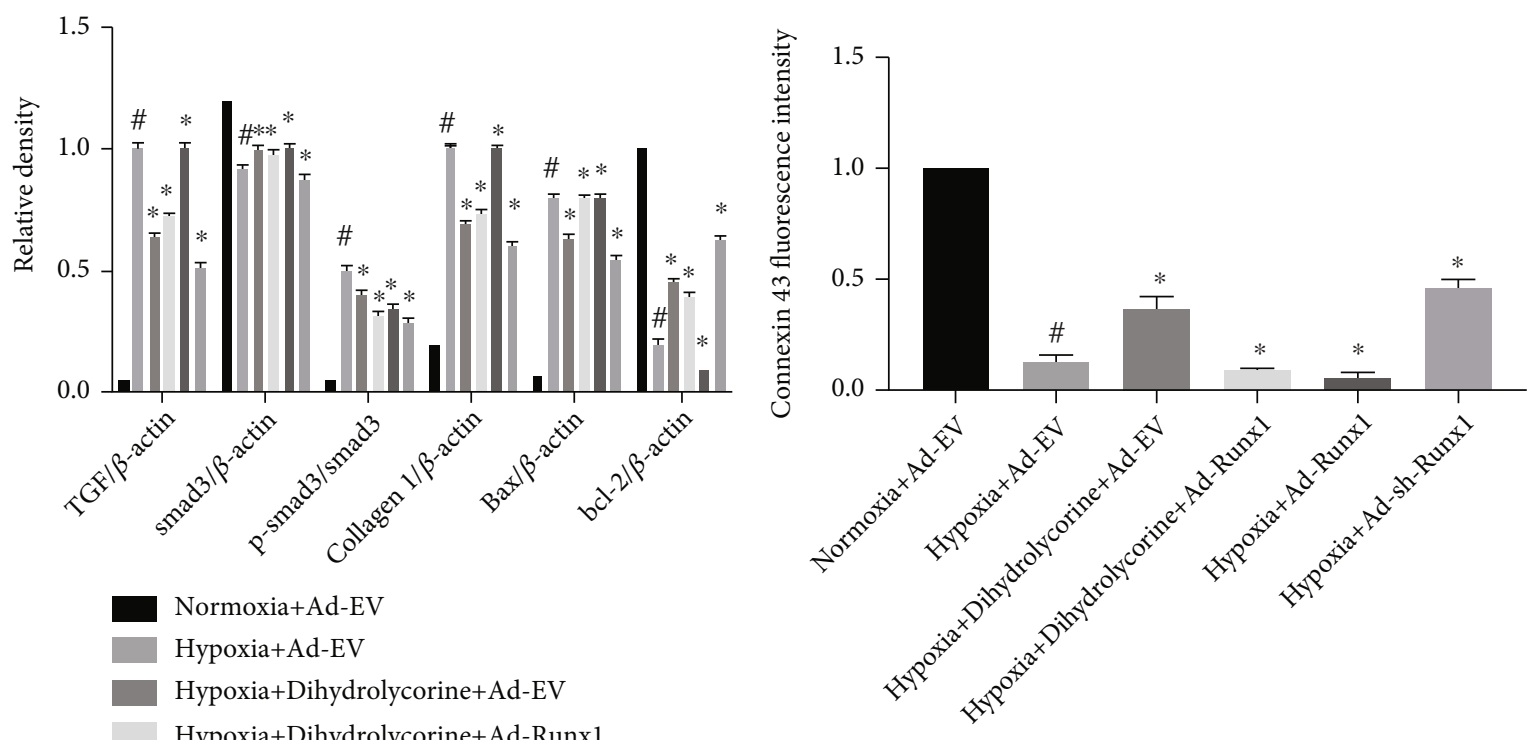

Hypoxia+Ad-EV

Hypoxia+Dihydrolycorine+Ad-EV

Hypoxia+Dihydrolycorine+Ad-Runx1

Hypoxia+Ad-Runx1

Hypoxia+Ad-sh-Runx1

(b)

(c)

Figure 6: Continued. 

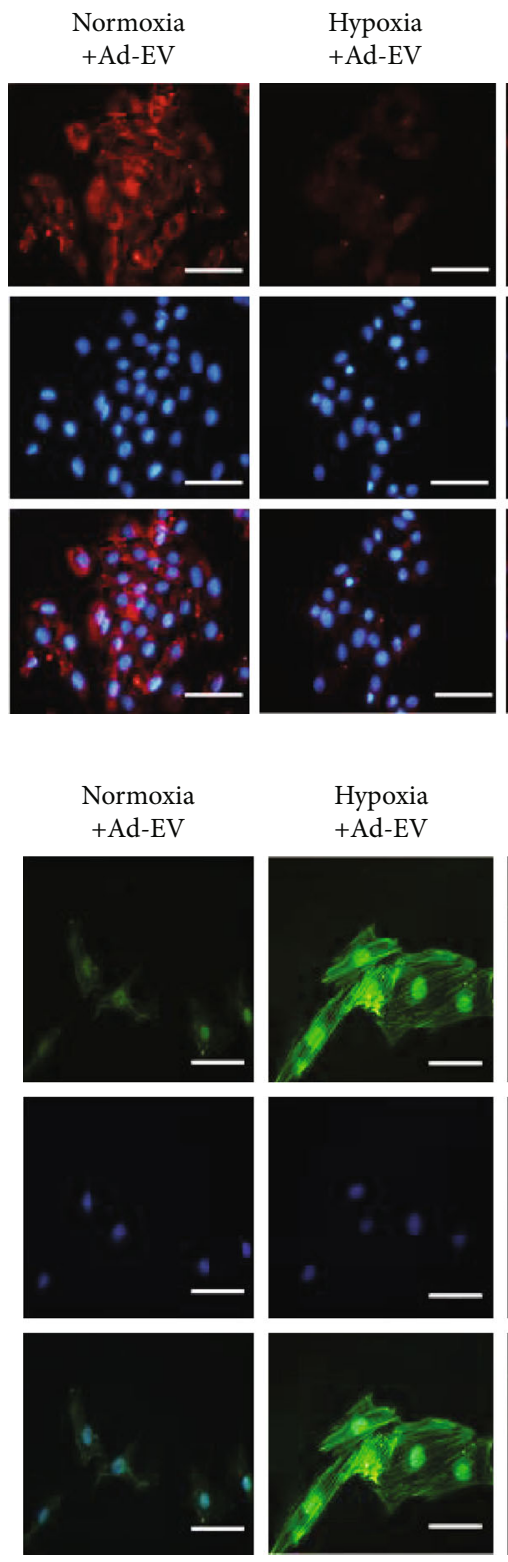
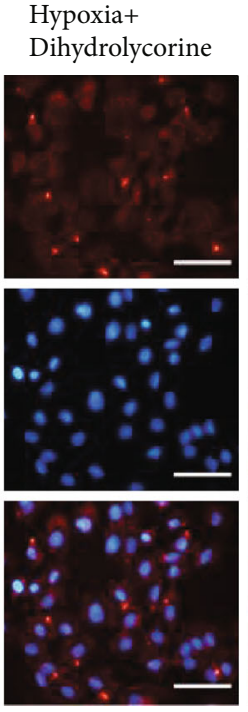

(d)

Hypoxia+

Dihy+Ad-Runx1
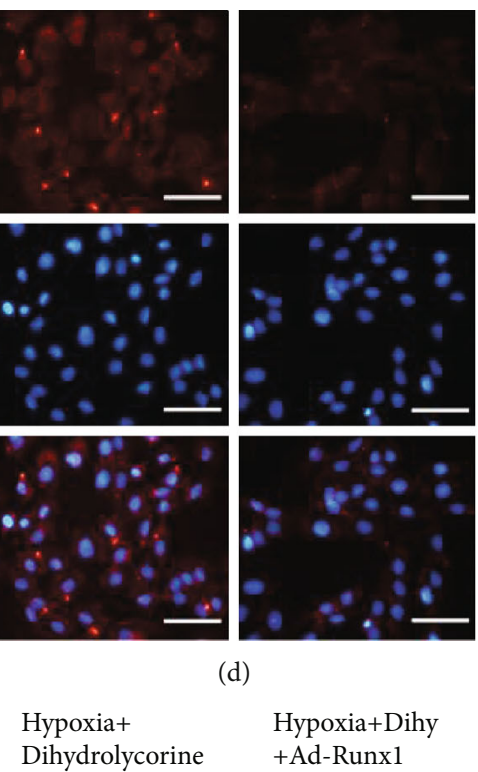

Hypoxia+Dihy +Ad-Runx1
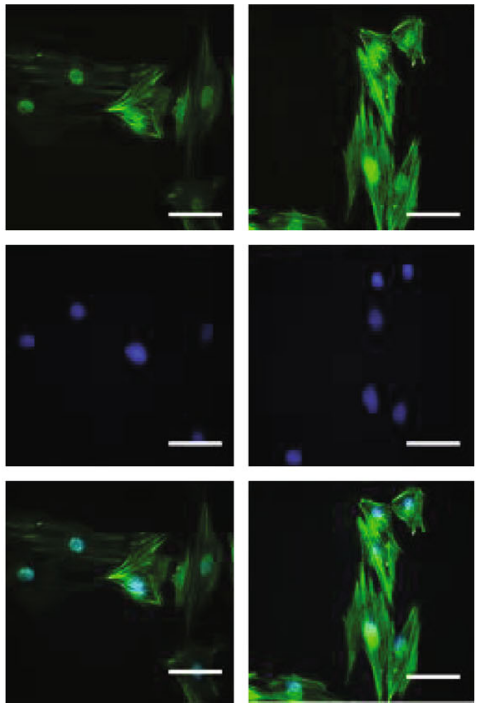
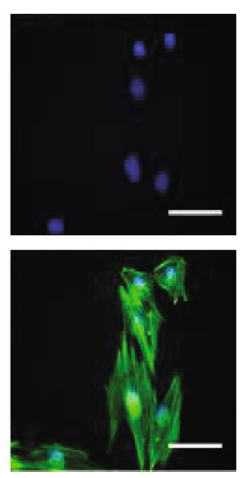

Hypoxia+
Ad-Runx1

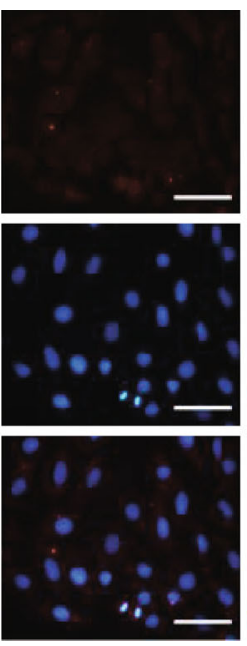

Hypoxia+ Ad-Runx
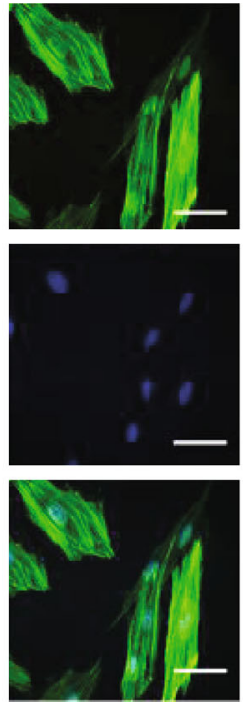

Hypoxia+ Ad-sh-Runx1

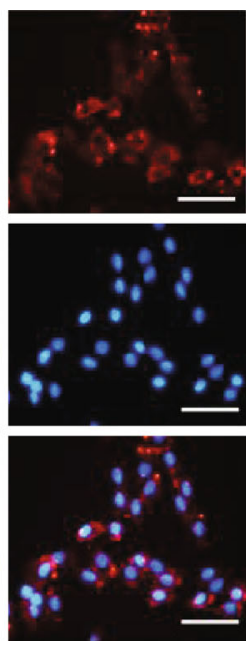

Hypoxia+ Ad-sh-Runx1
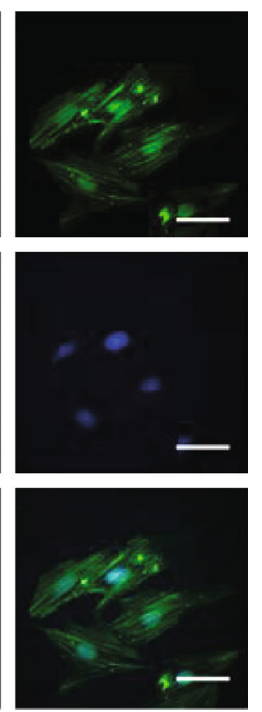

(e)

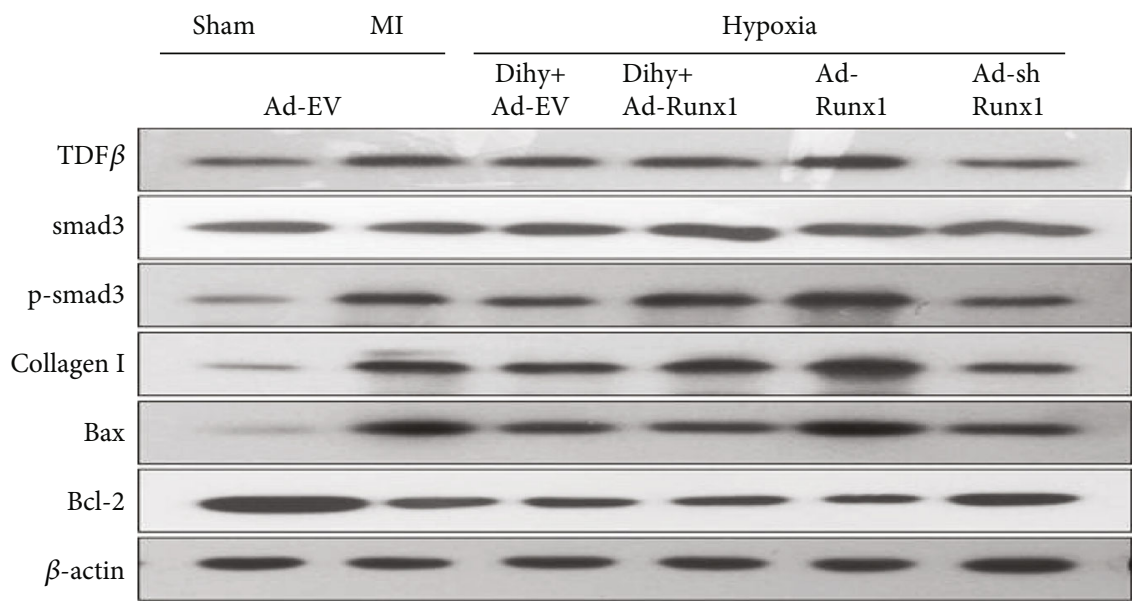

(f)

Figure 6: Continued. 


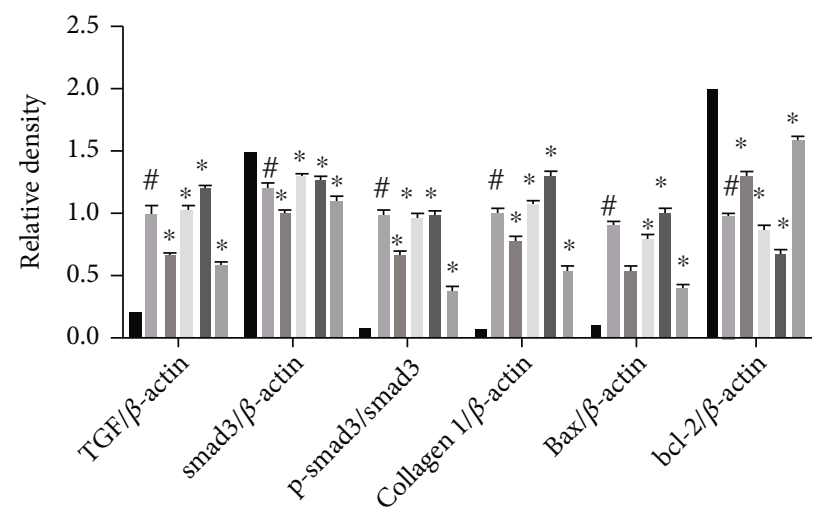

Sham+Ad-EV

MI+Ad-EV

MI+Dihydrolycorine+Ad-EV

MI+Dihydrolycorine+Ad-Runxl

MI+Ad-Runx 1

MI+Ad-sh-Runx1

(g)

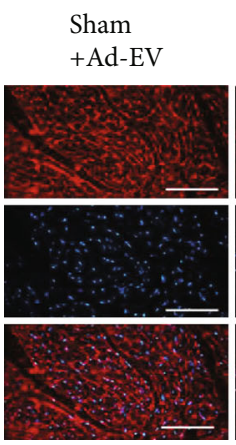

$$
\text { MI }
$$

+Ad-EV

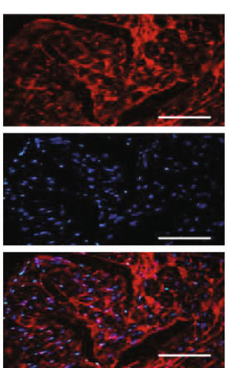

MI

+ Dihydrolycorine
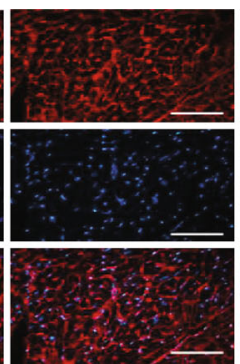

(h)

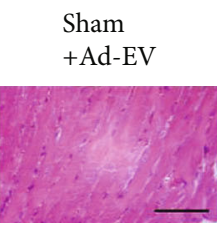

MI

+ Ad-EV

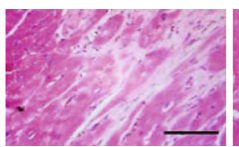

MI

+ Dihydrolycorine

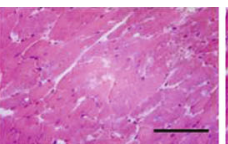

\section{MI+Dihy} +Ad-Runxl
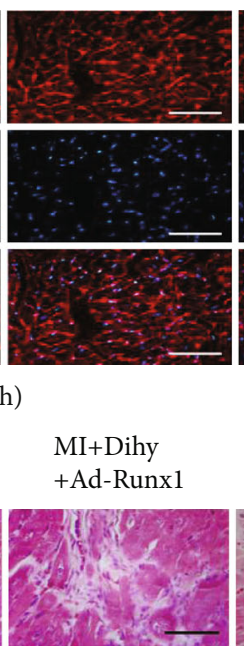

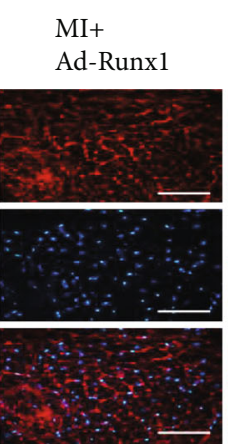

MI+ Ad-Runx1

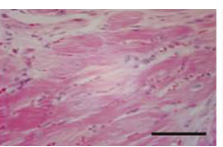

MI+

Ad-sh-Runx1

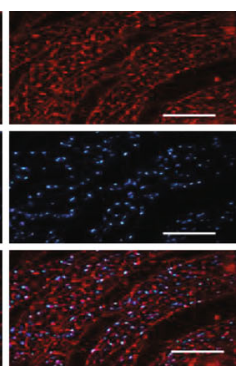

MI+ Ad-sh-Runx1

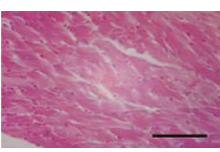

(i)

FIGURE 6: (a-e) Dihydrolycorine mitigated adverse cardiac remodeling after myocardial infarction (MI) reducing Runx1 in vivo and in vitro. Ad-EV, Ad-Runx1, and Ad-sh-Runx1 (10 mL/mL, MOI: $100: 1)$ were transfected into experimental cardiomyocytes for 6-8 hours, and these cells were treated with or without dihydrolycorine $(15 \mu \mathrm{M})$ under hypoxia conditions. MI rats $(n=8)$ and sham rats $(n=8)$ were injected with Ad-EV, Ad-Runx1, and Ad-sh-Runx1 (10 mL at each of four sites, $\left.1.2 \times 10^{10} \mathrm{PFU} / \mathrm{mL}\right)$ into the left ventricle free wall and then treated with or without dihydrolycorine $(20 \mathrm{mg} / \mathrm{kg})$. (a, b) Relative protein expression of collagen I, TGF $\beta$, smad3, p-smad3, Bax, and Bcl-2 according to western blotting. (c) Immunofluorescence staining of connexin 43 (red, magnification $\times 400)$. Scale bar $=50 \mu \mathrm{m}$. (d) Bar graphs of fluorescence intensity of connexin 43. (e) Immunofluorescence staining of $\alpha$-SMA (green, magnification $\times 400)$. Scale bar $=$ $50 \mu \mathrm{m}$. ( $\mathrm{f}-\mathrm{i}$ ) (f, g) Relative protein expression of collagen I, TGF $\beta$, smad3, p-smad3, Bax, and Bcl-2 according to western blotting. (h) Cardiac myocyte hypertrophy was evaluated using WGA (magnification $\times 100$ ) staining of left ventricular tissue from rats. Scale bar $=$ $100 \mu \mathrm{m}$. (i) Myocardial fibers in myocardial tissue sections were stained with hematoxylin and eosin (H\&E, magnification $\times 40)$. Scale bar $=200 \mu \mathrm{m}$. ${ }^{\#} P<0.05$ vs. normoxia and ${ }^{*} P<0.05$ vs. hypoxia; or ${ }^{\#} P<0.05$ vs. sham and ${ }^{*} P<0.05$ vs. MI; experiments were repeated more than three times.

indispensable role in this process. As shown in Figures 6(a) and 6(b) $(P<0.05)$, there was a significant downregulation of fibrotic genes and apoptosis genes in the Ad-sh-Runx1 thypoxia-treated cardiomyocytes and dihydrolycorine+hypoxia-treated cardiomyocytes. Administration of Ad-Runx1 sig- nificantly abolished the cardioprotective function of dihydrolycorine, as demonstrated by the increased expression of the cardiomyocyte fibrosis gene $\alpha$-SMA and decreased expression of punctuate gap junction protein connexin 43 in immunofluorescence staining (Figures 6(c)-6(e)). Moreover, 
TABLe 2: Dihydrolycorine fails to exert protective effects in MI rats injected with Ad-Runx1.

\begin{tabular}{lcccccc}
\hline Group $(n=8)$ & Sham+Ad-EV & MI+Ad-EV & MI+Dihy+Ad-EV & MI+Dihy+Ad-Runx1 & MI+Ad-Runx1 & MI+Ad-sh Runx1 \\
\hline LVEF (\%) & $79.26 \pm 2.13$ & $50.36 \pm 1.45$ & $64.16 \pm 2.64^{* \#}$ & $51.32 \pm 3.63^{* \#}$ & $48.41 \pm 2.61^{* \#}$ & $65.37 \pm 3.14^{* \#}$ \\
LVFS\% & $39.3 \pm 1.4$ & $21.5 \pm 3.2$ & $31.6 \pm 2.3^{* \#}$ & $21.9 \pm 4.1^{*}$ & $20.8 \pm 3.8^{* \#}$ & $31.9 \pm 2.6^{* \#}$ \\
LVESD (mm) & $2.4 \pm 0.26$ & $3.6 \pm 0.41$ & $2.9 \pm 0.35^{* \#}$ & $3.2 \pm 0.12^{* \#}$ & $3.8 \pm 0.32^{* \#}$ & $2.8 \pm 0.38^{* \#}$ \\
LVEDD (mm) & $3.7 \pm 0.4$ & $4.9 \pm 0.4$ & $4.0 \pm 0.4^{* \#}$ & $4.8 \pm 0.2^{\#}$ & $5.1 \pm 0.6^{* \#}$ & $3.9 \pm 0.3^{* \#}$ \\
E/A ratio & $2.14 \pm 0.36$ & $1.48 \pm 0.45$ & $1.83 \pm 0.48^{\#}$ & $1.46 \pm 0.61^{* \#}$ & $1.42 \pm 0.21^{* \#}$ & $1.86 \pm 0.36^{*}$ \\
HR (bmp) & $520 \pm 8$ & $480 \pm 5$ & $502 \pm 5^{* \#}$ & $478 \pm 4^{* \#}$ & $469 \pm 9^{*}$ & $506 \pm 7^{* \#}$ \\
\hline
\end{tabular}

Values are mean $\pm \mathrm{SD}, P<0.05$. The data are presented as means and SD. ${ }^{*} P<0.05$ versus sham $+\mathrm{Ad}-\mathrm{EV} ;{ }^{*} P<0.05$ versus $\mathrm{MI}+\mathrm{Ad}-\mathrm{EV}$. LVEDD: left ventricular end-diastolic dimension; LVESD: left ventricular end-systolic dimension; LVEF: left ventricular ejection fraction; LVFS: left ventricular systolic function; HR: heart rate.

Ad-Runx1, which was injected into the left ventricle of MI rats, could offset the cardioprotective effect of dihydrolycorine, as characterized by increased in LVEDD and LVESD and decreased LVEF and LVFS compared to the MI+dihydrolycorine+Ad-EV group (Table 2). As expected, the stimulation of Runx1 in MI rats failed to rescue myocardial fibrosis as measured by the expression of fibrotic and apoptotic genes (Figures 6(f) and 6(g)) and was unable to mitigate cardiomyocyte hypertrophy even after dihydrolycorine treatment (Figure 6(h)). H\&E staining confirmed that in the MI+AdRunxl group and the MI+dihydrolycorine+Ad-Runxl group, irregular myocardial fibers and obscure intercellular borders were trapped. In comparison to the MI+dihydrolycorine group or the MI+Ad-sh-Runxl group, the cardiac fibers were rearranged. Taken together, these results confirm Runx1 directly influences the cardioprotective effect of dihydrolycorine in hypoxia-treated cardiomyocytes and MI rats.

\section{Discussion}

MI subsequently triggers necrotic myocardium and myocardial fibrosis due to hypoxia caused by sudden blood flow occlusion of the coronary artery [31]. This, in turn, induces excessive and progressive remodeling of the cardiac infarct tissue and remote myocardium, ultimately leading to ventricular dysfunction and the clinical syndrome of heart failure $[32,33]$. Depending on the application of early percutaneous coronary intervention, premature mortality can dramatically decrease [34]. Unfortunately, the incidence of heart failure and rehospitalization after MI has increased in recent decades because this surgery only reduces symptoms $[35,36]$. However, the underlying mechanisms of adverse cardiac remodeling after MI remain unclear. Furthermore, no specific therapy is available to cure or alleviate adverse cardiac remodeling after MI. In this study, we identified the cardioprotective effect of dihydrolycorine against adverse cardiac remodeling after MI and found that Runx1 is a critical regulator of myocardial fibrosis in the occurrence and development of adverse cardiac remodeling.

Recently, several studies have reported that Runx1 may be a promising therapeutic target for cardiovascular diseases [23, 37, 38]. Runx1 directly targets phosphatidylcholine transfer proteins in patients with cardiovascular diseases [39]. Runx1 induces endogenous heart repair by controlling the regenerative response of zebrafish cardiac cells [40]. Initially, we determined that Runxl was significantly upregulated and was the most differentially expressed MI-related gene after MI surgery based on MI RNA-seq data obtained from the GSE1146395 dataset from GEO (Figures 2(a)$2(\mathrm{~d}))$. Consistent with this, western blotting revealed that Runx1 expression increased after MI (Figures 2(e) and 2(f)). However, the ability of Runxl to function as a regulator of adverse cardiac remodeling after MI remains unknown.

It has been previously reported that dihydrolycorine exerts a positive effect on adverse cardiac remodeling after MI, and the Natural Compound Library Screening identified dihydrolycorine as a candidate drug against cardiac fibrosis, as reported by Schimmel et al. in 2020 [14]; however, its ability to attenuate cardiac fibrosis after MI remains unknown. Surprisingly, we found that dihydrolycorine could rescue cardiac function after $\mathrm{MI}$, as observed by increased LVEF and LVFS and decreased LVEDD and LVESD (Table 1). Moreover, fibrotic genes such as collagen I, TGF $\beta$, and $\mathrm{p}$-smad 3 and the apoptosis gene Bax were downregulated in the left ventricles of dihydrolycorine-treated MI rats (Figures 3(c) and 3(d)), thus preventing cardiomyocytes from becoming hypertrophic (Figure 3(a)) and irregular myocardial fibers and obscure intercellular borders in infarct areas (Figure 3(b)). Interestingly, there is decreased expression of Runx1 in the left ventricle tissues of dihydrolycorine-treated MI rats (Figures 3(e) and 3(f)).

Subsequently, we also demonstrated that Runx1 is the binding protein for dihydrolycorine through biotinylated protein interaction pull-down assays (Figures 4(a)-4(d)) and molecular docking analyses (Figures 4(e)-4(g)). Moreover, we observed that long-term hypoxia led to the upregulation of fibrotic and apoptotic gene expression in cardiomyocytes, which could be attenuated by treatment with dihydrolycorine (Figures 5(b) and 5(c)). Disruption of $\alpha$-SMA and the augmentation of connexin 43 , as shown by immunofluorescence staining results, in dihydrolycorinetreated hypoxia-cardiomyocytes indicated a reduction in myocardial fibrosis when compared with the hypoxiacardiomyocyte group. As expected, Runx1 levels were lower in dihydrolycorine-treated hypoxia-cardiomyocytes than in the control group (Figures 5(d) and 5(e)). Finally, we found that stimulation of Runx1 in dihydrolycorine-treated hypoxia-cardiomyocytes abolished the cardioprotective 


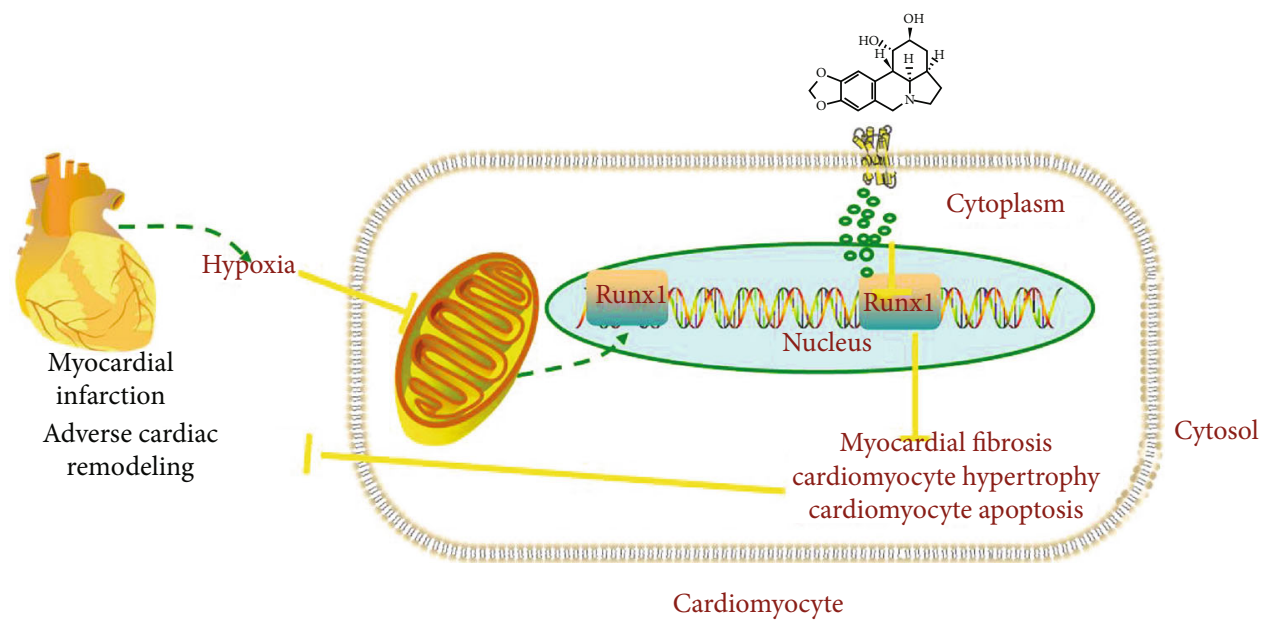

FIGURE 7: Schematic illustration indicating the cardioprotective effects of dihydrolycorine on myocardial infarction (MI) induced adverse cardiac remodeling.

effect of dihydrolycorine, as demonstrated by the increased expression of fibrotic and apoptotic genes (Figures 6(a) and $6(\mathrm{~b}))$, the augmentation of $\alpha$-SMA, and reduction of connexin 43 (Figures 6(c)-6(e)) compared with the hypoxia + dihydrolycorine+Ad-EV group. Concurrently, Runx1 stimulation counteracted the cardioprotective effects of dihydrolycorine, as demonstrated by a decrease in LVEF and LVFS, an increase in LVEDD and LVESD, and aggravation of cardiomyocyte hypertrophy (Figures 6(g) and 6(h)) and irregular myocardial fibers and obscure intercellular borders in infarct areas (Figure 6(i)) compared with the MI+dihydrolycorine + Ad-EV group (Table 2).

\section{Conclusions}

In summary, in the present study, we found that dihydrolycorine could alleviate myocardial fibrosis progression, leading to adverse cardiac remodeling after MI. This was associated with decreased expression of Runx1 (Figure 7). Thus, this study demonstrated that dihydrolycorine might be a promising agent for cardiac antifibrotic therapy after MI.

\section{Data Availability}

MI RNA-seq data based on the GSE1146395 [27] dataset was acquired from the Gene Expression Omnibus (GEO) database (https://www.ncbi.nlm.nih.gov/geo/). Other data are available upon request (contact the corresponding author).

\section{Ethical Approval}

All experiments and procedures revolving in animals in this study were performed with humanitarian care in accordance with the Institutes of the First Affiliated Hospital of Wenzhou Medical University Health Guidelines for Laboratory Animals.

\section{Conflicts of Interest}

The authors have no conflict of interest to declare.

\section{Authors' Contributions}

Tingjuan Ni, Xingxiao Huang, Sunlei Pan, and Zhongqiu Lu conceived and designed the study. Tingjuan $\mathrm{Ni}$, Xingxiao Huang, and Sunlei Pan conducted experiments and analyzed the data. Tingjuan Ni and Xingxiao Huang drafted the manuscript. Sunlei Pan and Zhongqiu Lu revised the draft critically. All authors reviewed and approved the final manuscript.

\section{Acknowledgments}

This work was supported by the National Natural Science Foundation of China (No. 81772112).

\section{References}

[1] W. C. Members, M. J. B. B. Clyde, and W. Yancy, "2016 ACC/AHA/HFSA focused update on new pharmacological therapy for heart failure: an update of the 2013 ACCF/AHA guideline for the management of heart failure," Circulation, vol. 13, no. 134, pp. e282-e293, 2016.

[2] P. Lv, C. Li, M. Wang, J. Ren, Y. Zhang, and G. Fu, "TANKbinding kinase 1 alleviates myocardial ischemia/reperfusion injury through regulating apoptotic pathway," Biochemical and Biophysical Research Communications, vol. 528, no. 3, pp. 574-579, 2020.

[3] K. D. P. A. Konstam Ma, "Left ventricular remodeling in heart failure: current concepts in clinical significance and assessment," JACC: Cardiovascular Imaging, vol. 1, no. 4, pp. 98$108,2011$.

[4] H. Chen, A. Moreno-Moral, F. Pesce et al., "Author Correction: WWP2 regulates pathological cardiac fibrosis by modulating SMAD2 signaling," Nature Communications, vol. 10, no. $1,2019$. 
[5] G. Chen, X. Song, D. Lin, and P. Xu, "Isofraxidin alleviates myocardial infarction through NLRP3 inflammasome inhibition," INFLAMMATION, vol. 43, no. 2, pp. 712-721, 2020.

[6] K. Takeda, "The stereochemistry of dihydrolycorine," Pharmaceutical Bulletin, vol. 3, no. 5, pp. 234-240, 1957.

[7] R. E. Hayden, G. Pratt, M. T. Drayson, and C. M. Bunce, "Lycorine sensitizes CD40 ligand-protected chronic lymphocytic leukemia cells to bezafibrate- and medroxyprogesterone acetate-induced apoptosis but dasatanib does not overcome reported CD40-mediated drug resistance," Haematologica, vol. 95, no. 11, pp. 1889-1896, 2010.

[8] S. Wu, Y. Qiu, Y. Shao et al., "Lycorine displays potent antitumor efficacy in colon carcinoma by targeting STAT3," Frontiers in Pharmacology, vol. 9, p. 881, 2018.

[9] M. Roy, L. Liang, X. Xiao et al., "Lycorine downregulates HMGB1 to inhibit autophagy and enhances bortezomib activity in multiple myeloma," Theranostics, vol. 6, no. 12, pp. 2209-2224, 2016.

[10] N. Cortes, R. A. Posada-Duque, R. Alvarez et al., "Neuroprotective activity and acetylcholinesterase inhibition of five Amaryllidaceae species: a comparative study," Life Sciences, vol. 122, pp. 42-50, 2015.

[11] S. Chen, G. Jin, K. Huang et al., "Lycorine suppresses RANKLinduced osteoclastogenesis _in vitro_and prevents ovariectomyinduced osteoporosis and titanium particle-induced osteolysis _in vivo_”S Scientific Reports, vol. 5, no. 1, 2015.

[12] J. Liu, Y. Yang, Y. Xu, C. Ma, C. Qin, and L. Zhang, "Lycorine reduces mortality of human enterovirus 71-infected mice by inhibiting virus replication," Virology Journal, vol. 8, no. 1, p. 483, 2011.

[13] G. S. Çitoğlu, Ö. B. Acıkara, B. S. Yılmaz, and H. Özbek, "Evaluation of analgesic, anti-inflammatory and hepatoprotective effects of lycorine from Sternbergia fisheriana (Herbert) Rupr," Fitoterapia, vol. 83, no. 1, pp. 81-87, 2012.

[14] K. Schimmel, M. Jung, A. Foinquinos et al., "Natural compound library screening identifies new molecules for the treatment of cardiac fibrosis and diastolic dysfunction," CIRCULATION, vol. 141, no. 9, pp. 751-767, 2020.

[15] S. Bäckström, M. Wolf-Watz, C. Grundström, T. Härd, T. Grundström, and U. H. Sauer, "The RUNX1 runt domain at $1.25 \AA$ resolution: a structural switch and specifically bound chloride ions modulate DNA binding," Journal of Molecular Biology, vol. 322, no. 2, pp. 259-272, 2002.

[16] K. Blyth, E. R. Cameron, and J. C. Neil, "The runx genes: gain or loss of function in cancer," Nature Reviews. Cancer, vol. 5, no. 5, pp. 376-387, 2005.

[17] X. Zhang, S. Ma, R. Zhang et al., "Oncostatin M-induced cardiomyocyte dedifferentiation regulates the progression of diabetic cardiomyopathy through B-Raf/Mek/Erk signaling pathway," Acta Biochimica et Biophysica Sinica, vol. 48, no. 3, pp. 257$265,2016$.

[18] G. Lluri, V. Huang, M. Touma, X. Liu, A. W. Harmon, and A. Nakano, "Hematopoietic progenitors are required for proper development of coronary vasculature," Journal of Molecular and Cellular Cardiology, vol. 86, pp. 199-207, 2015.

[19] N. Martinez-Soria, L. McKenzie, J. Draper et al., "The oncogenic transcription factor RUNX1/ETO corrupts cell cycle regulation to drive leukemic transformation," Cancer Cell, vol. 34, no. 4, pp. 626-642.e8, 2018.

[20] Y. Ochi, A. Kon, T. Sakata et al., "Combined cohesin-RUNX1 deficiency synergistically perturbs chromatin looping and causes myelodysplastic syndromes," Cancer Discovery, vol. 10, no. 6, pp. 836-853, 2020.

[21] T. Zhen, Y. Cao, G. Ren et al., "RUNX1 and CBF $\beta$-SMMHC transactivate target genes together in abnormal myeloid progenitors for leukemia development," BLOOD, vol. 136, no. 21, pp. 2373-2385, 2020.

[22] C. S. McCarroll, W. He, K. Foote et al., "Runx1 deficiency protects against adverse cardiac remodeling after myocardial infarction," Circulation, vol. 137, no. 1, pp. 57-70, 2018.

[23] A. Riddell, M. McBride, T. Braun et al., "RUNX1: an emerging therapeutic target for cardiovascular disease," Cardiovascular Research, vol. 116, no. 8, pp. 1410-1423, 2020.

[24] T. Ni, N. Lin, X. Huang et al., "Icariin ameliorates diabetic cardiomyopathy through apelin/Sirt3 signalling to improve mitochondrial dysfunction," Frontiers in Pharmacology, vol. 11, 2020.

[25] X. Hou, H. Zeng, Q. Tuo, D. Liao, and J. Chen, “Apelin gene therapy increases autophagy via activation of sirtuin 3 in diabetic heart," Diabetes Research - Open Journal, vol. 1, no. 4, pp. 84-91, 2015.

[26] L. Hu, M. Ding, D. Tang et al., “Targeting mitochondrial dynamics by regulating Mfn2 for therapeutic intervention in diabetic cardiomyopathy," Theranostics, vol. 9, no. 13, pp. 3687-3706, 2019.

[27] M. Merentie, L. Lottonen-Raikaslehto, V. Parviainen et al., "Efficacy and safety of myocardial gene transfer of adenovirus, adeno- associated virus and lentivirus vectors in the mouse heart," Gene Therapy, vol. 23, no. 3, pp. 296-305, 2016.

[28] T.-J. Park, J. H. Park, G. S. Lee et al., "Quantitative proteomic analyses reveal that GPX4 downregulation during myocardial infarction contributes to ferroptosis in cardiomyocytes," Cell Death \& Disease, vol. 10, no. 11, pp. 815-835, 2019.

[29] R. Janardhanan, S. Kilari, E. B. Leof, and S. Misra, "Hyperglycemia-induced modulation of the physiognomy and angiogenic potential of fibroblasts mediated by matrix metalloproteinase2: implications for venous stenosis formation associated with hemodialysis vascular access in diabetic milieu," Journal of Vascular Research, vol. 52, no. 5, pp. 334-346, 2016.

[30] T. Ni, N. Lin, W. Lu et al., "Dihydromyricetin prevents diabetic cardiomyopathy via miR-34a suppression by activating autophagy," CARDIOVASCULAR DRUGS AND THERAPY, vol. 34, no. 3, pp. 291-301, 2020.

[31] W. Yu, S. Sun, H. Xu, C. Li, J. Ren, and Y. Zhang, "TBC1D15/RAB7-regulated mitochondria-lysosome interaction confers cardioprotection against acute myocardial infarction-induced cardiac injury," Theranostics, vol. 10, no. 24, pp. 11244-11263, 2020.

[32] L. K. Newby, "Inflammation as a treatment target after acute myocardial infarction," The New England Journal of Medicine, vol. 381, no. 26, pp. 2562-2563, 2019.

[33] X. Wang, Z. Guo, Z. Ding, and J. L. Mehta, "Inflammation, autophagy, and apoptosis after myocardial infarction," Journal of the American Heart Association, vol. 7, no. 9, 2018.

[34] C. Peet, A. Ivetic, D. I. Bromage, and A. M. Shah, "Cardiac monocytes and macrophages after myocardial infarction," CARDIOVASCULAR RESEARCH, 2020.

[35] J. Chen, A. F. Hsieh, K. Dharmarajan, M. Frederick, A. Masoudi, and H. M. Krumholz, "National trends in heart failure hospitalization after acute myocardial infarction for Medicare beneficiaries 1998-2010," CIRCULATIONAHA, vol. 24, no. 128, pp. 2577-2584, 2013. 
[36] R. S. V. M. Levy, "Heart failure long-term trends in the incidence of heart failure after myocardial infarction," Circulation, vol. 118, pp. 2057-2062, 2008.

[37] S. H. Lee, S. Manandhar, and Y. M. Lee, "Roles of RUNX in hypoxia-induced responses and angiogenesis," in Advances in Experimental Medicine and Biology, pp. 449-469, Springer, Singapore, 2017.

[38] X. Li, S. Zhang, M. Wa, Z. Liu, and S. Hu, "MicroRNA-101 protects against cardiac remodeling following myocardial infarction via downregulation of Runt-related transcription factor 1," Journal of the American Heart Association, vol. 8, no. 23, p. e13112, 2019.

[39] G. Mao, N. Songdej, D. Voora et al., "Transcription factor RUNX1 regulates PlateletPCTP(Phosphatidylcholine transfer protein): implications for cardiovascular Events," Circulation, vol. 136, no. 10, pp. 927-939, 2017.

[40] J. K. X. W. Jefferson, "Runx1 promotes scar deposition and inhibits myocardial proliferation and survival during zebrafish heart regeneration," DEVELOPMENT, vol. 8, no. 147, p. v186569, 2020. 